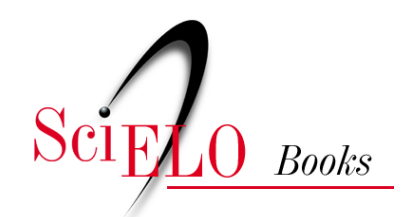

\title{
Capítulo III - Evaluación de la cosecha de neblina como una fuente potencial para el aprovechamiento de agua
}

\author{
Christian Domínguez \\ Paola Echeverría \\ Marcos Villacís \\ Sophie Violette
}

\section{SciELO Books / SciELO Livros / SciELO Libros}

DOMÍNGUEZ, C., ECHEVERRÍA, P., VILLACÍS, M., and VIOLETTE, S. Evaluación de la cosecha de neblina como una fuente potencial para el aprovechamiento de agua. In: INGA ORTEGA, E., ed. Aplicaciones e innovación de la ingeniería en ciencia y tecnología [online]. Quito: Editorial AbyaYala, 2019, pp. 97-142. ISBN: 978-9978-10-491-0. https://doi.org/10.7476/9789978104910.0005.

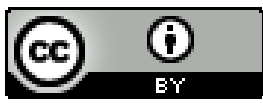

All the contents of this work, except where otherwise noted, is licensed under a Creative Commons Attribution 4.0 International license.

Todo o conteúdo deste trabalho, exceto quando houver ressalva, é publicado sob a licença Creative Commons Atribição $\underline{4.0}$.

Todo el contenido de esta obra, excepto donde se indique lo contrario, está bajo licencia de la licencia $\underline{\text { Creative }}$ Commons Reconocimento 4.0. 


\title{
CAPÍTULO III
}

\section{Evaluación de la cosecha de neblina como una fuente potencial para el aprovechamiento de agua}

\section{Evaluation of fog water collection as a potential source of water supply}

\author{
Christian Domínguez*, Paola Echeverría ${ }^{\S}$, Marcos Villacís ${ }^{\dagger}$, Sophie Violette ${ }^{\top}$ \\ *Universidad Politécnica Salesiana Ecuador, §† Escuela Politécnica Nacional Ecua- \\ dor, TUniversidad Sorbona Francia \\ "email: cdominguez@ups.edu.ec \\ §email: pao_echev1@hotmail.com \\ †email: marcos.villacis@epn.edu.ec \\ †email:sophie.violette@ens.fr
}

\section{Resumen}

El término "ciudades inteligentes" es asociado únicamente con el empleo de tecnologías de la información en la infraestructura de una ciudad. Sin embargo, un aspecto fundamental para satisfacer las necesidades de una población es el aprovechamiento de recursos naturales, entre ellos el agua. En este sentido, las técnicas de aprovechamiento de agua deben ser sostenibles y amigables con el ambiente, este puede ser el caso de la cosecha de neblina. En este estudio, se presenta una metodología para cuantificar la intercepción de neblina por un sistema de captación y evaluar su potencial como una fuente de agua alternativa. Esta metodología fue probada en un sitio experimental en la parte alta de la isla San Cristóbal (Galápagos). Se realizó un monitoreo de dos meses que incluyó la medición de las principales variables meteorológicas y la intercepción de neblina. Se utilizaron tres colectores de neblina, un cilíndrico (CFC), y dos colectores estándar de 35\% (SFC35) y 50\% (SFC50) de coeficiente de sombra, respectivamente. Dado que los colectores captan tanto neblina como lluvia, se utilizó un modelo geométrico para separar estas dos variables. Los resultados de este estudio muestran que los colectores CFC, SFC35 y SFC50 tienen un rendimiento de 3.7, 4.1 y $4.8 \mathrm{~mm} /$ día, respectivamente. El colector SFC50 es el que tiene un mejor rendimiento de intercepción de neblina, 
el que puede ser atribuido a su alta eficiencia aerodinámica comparada con los otros. Al analizar las variables meteorológicas se evidencia que el potencial de captura de neblina puede ser aún mayor.

\section{Palabras clave}

Ciudades inteligentes, clima, galápagos, intercepción de neblina, sostenibilidad

\section{Abstract}

The term "smart cities" is usually associate to the use of information technologies in a city infrastructure. Nevertheless, the use of natural resources, such as water, is an important aspect to satisfy the inhabitants necessities. In this sense, techniques to supply water must be sustainable and environmentally friendly, which is the case of fog water collection. In this study, we present a methodology to quantify fog water intercepted by a fog gauge system and to assess its potential as a source of water supply. This methodology is tested in an experimental site located at the highlands of San Cristobal Island (Galapagos), which included two months monitoring of the main meteorological variables and fog interception. Three fog gauges were used to quantify fog interception, a cylindrical (CFC) and two standards of 35\% (SFC35) and 50\% (SFC50) shading coefficient, respectively. Given that fog gauge collectors capture both fog and rainfall, we used a geometrical model to separate both variables. Results show that collectors' CFC, SFC35 and SFC50 have an average performance of 3.7, 4.1 and $4.8 \mathrm{~mm} /$ day, respectively. SFC50 collector presents the best performance which can be related to its relative higher aerodynamic efficiency. The analysis of the meteorological variables evidences that fog water collection potential can be even higher at this site.

\section{Keywords}

Smart cities, climate, Galapagos, fog water interception, sustainability

\section{Introducción}

En los últimos años, el término "ciudades inteligentes" se ha utilizado con mayor frecuencia en la política de gobiernos y municipios, en artículos científicos, e inclusive en medios de comunicación masivos y redes sociales. Esta popularidad se alcanzó, debido al constante debate de cómo las nuevas tecnologías podrían ayudar a mejorar la habitabilidad en áreas urbanas.

No existe un concepto claro sobre qué son las ciudades inteligentes, debido a que su definición depende de la visión que se tenga de las mismas [1]. Mientras varios autores relacionan este concepto con el empleo de tecnologías de la información en la infraestructura 
de una ciudad o al desarrollo de negocios (ej. [2]-[7] otros autores orientan este concepto hacia el bienestar y desarrollo de sus habitantes (ej. [8]-[11]) , y pocos hacia el tema ambiental (ej. [12]-[14]), como el manejo adecuado de los recursos naturales. Tanto Albino et al., 2015 [1] y Batisha, 2015 [15] concluyen que dicho concepto es único para cada ciudad y depende de las necesidades de la misma.

Sin duda alguna, una de las necesidades más importantes de toda ciudad, es el aprovechamiento de recursos naturales, entre ellos el agua. Además de que el agua es un elemento fundamental para la supervivencia de los seres vivos, también es un factor importante en los procesos de producción, para el desarrollo económico de una población e inclusive para la recreación de sus habitantes. Sin embargo, muchas regiones en el mundo sufren de falta de agua [16]-[18]. En al año 2000, de 1.2 a 2.4 billones de personas (20 al 39\% de la población mundial) vivía en cuencas hidrográficas expuestas a falta de recursos hídricos [19]-[24]. Tanto el incremento de la población y el crecimiento del desarrollo económico global, son factores importantes para el crecimiento de la escasez del agua de los habitantes. También, el cambio climático constituye un factor clave para la evaluación de la disponibilidad de recursos hídricos, cambios en la temperatura y en los patrones de precipitación resultan una amenaza para el abastecimiento de agua en varios sitios. Varios autores [16], [18]-[25] han utilizado diferentes modelos hidrológicos y modelos climáticos globales con diversos posibles escenarios para estimar la disponibilidad de agua en años futuros, se espera que para el año 2050, entre el 37 y 53\% de población global viva en regiones con problemas de falta de agua. En la actualidad, muchos países ya están tomando conciencia de este asunto. Países como Australia [26], China [27], España [28], [29] y Estados Unidos [30], ya están contemplando medidas y se encuentran buscando alternativas para asegurar el abastecimiento de sus poblaciones. En este contexto, una ciudad inteligente debe tomar en cuenta el problema de fuentes de agua para el suministro de sus habitantes.

En la actualidad, el abastecimiento de agua para las poblaciones proviene de dos fuentes principales: i) el agua superficial de ríos y lagunas, conocida como "agua azul", en donde el agua es tomada a través de infraestructura hidráulica; y ii) el agua subterránea, 
conocida como "agua verde", en donde el agua es extraída de acuíferos mediante el bombeo de pozos y usos de galerías. Es claro que las poblaciones que se ubican cerca de fuentes superficiales de agua pueden aprovechar este recurso. Sin embargo, no se tiene certeza sobre el efecto que tendría el cambio climática en la disponibilidad de las misma [18], dicha incertidumbre podría contemplar una disminución en su cantidad. Las poblaciones que se benefician de aguas subterráneas tienen la dificultad de que estas fuentes son muy susceptibles a la contaminación o a la intrusión del agua del mar (en el caso de zonas costeras), además de la incertidumbre en cuanto al cambio climático (existe la posibilidad que las precipitaciones sean más intensas y esporádicas, generando mayor escorrentía y posiblemente reduciendo la recarga de acuíferos). Existen otras fuentes de agua alternativa como: el reciclamiento de aguas residuales, la desalinización de agua del mar o el aprovechamiento de la neblina, este último conocido como "cosecha de neblina". Las dos primeras alternativas, aunque resulten en fuentes prácticamente ilimitadas de agua, su potabilización requiere de una cantidad considerable de energía, y actualmente se utiliza combustibles fósiles en su mayoría [28]; motivo por el cual estos sistemas no podrían ser considerados como sustentables. La cosecha de neblina, podría resultar en una fuente de agua sustentable, el equipamiento y mantenimiento es sencillo y relativamente barato. Este sistema se lo ha utilizado en pequeñas comunidades con resultados satisfactorios [15], [31], [32]. El problema de la cosecha de agua es que está limitado a zonas expuestas a neblina y que la cantidad potencial de agua recolectada por este sistema es desconocida hasta cierto punto.

La neblina consiste en pequeñas gotas de agua que se encuentran en suspensión debido a que su tamaño no es lo suficientemente grande para que su velocidad de caída supere la velocidad de empuje del aire [33], permitiéndolas precipitarse. La neblina se puede formar por diversos procesos físicos en la atmósfera [34], [35], pero en general ocurre debido a la condensación de la evaporación de la humedad del suelo, vegetación, de masas de agua o de masas de aire húmedas. Cuando la neblina es llevada por el viento, las pequeñas gotas de agua chocan con la estructura de la vegetación, estas coalescen formando gotas más grandes que finalmente se precipitan 
en el suelo [33], [34], [36]. A este proceso se conoce como intercepción de neblina, y puede ser una fuente importante de agua para varios ecosistemas, alcanzando valores entre 4 y $98 \%$ del aporte de la precipitación [37]-[43].

Los habitantes de regiones con escasez de fuentes de agua convencionales han tratado de aprovechar este proceso hidrológico como una fuente de agua alternativa, al utilizar colectores para captar neblina. Existen varios proyectos de sistemas de colección de neblina alrededor del mundo (Chile, Ecuador, Sudáfrica, Namibia, Omán, Arabia Saudita, España). De acuerdo a la literatura, la producción de diferentes colectores de neblina oscila entre 2 y 10 $1 / \mathrm{m}^{2}$ día en promedio [15], [31], [51], [52], [32], [44]-[50], alcanzando valores de hasta $300 \mathrm{l} / \mathrm{m}^{2}$.día [45]. De acuerdo a Batisha, 2007 [15], la implementación de estos sistemas puede resultar atrayente debido a que se puede colectar una gran cantidad de agua, su costo es relativamente bajo o comparable con otros sistemas de agua, los colectores son simples y no requieren energía externa, y porque el agua captada se espera que sea de buena calidad. Sin embargo, la factibilidad de este sistema depende de un factor importante como es la cantidad de neblina captable. Antes de la implementación de este tipo de sistema se debe realizar una investigación sobre la cuantificación de neblina en el sitio con diferentes colectores de neblina y que incluya el monitoreo de otras variables meteorológicas que permitan conocer las condiciones que favorecen la intercepción de neblina. Debido a que estos sistemas captan tanto lluvia como neblina, sería también importante separar estas componentes para evaluar realmente el aporte de la neblina.

Conociendo la problemática del incremento de la demanda de agua, uno de los componentes que se tiene que trabajar alrededor de una ciudad inteligente es el manejo y aprovechamiento de los recursos hídricos. Sin duda alguna, los sistemas de aprovechamiento de agua deberían ser sustentables, con tecnologías que sean amigables con el ambiente y que incluya la participación de la población. Uno de estos sistemas puede ser la captación de neblina. En este trabajo presentamos una metodología simple para evaluar la cantidad de neblina recolectada por un sistema de captación de neblina, determinando no solamente la cantidad de neblina captada sino también identificando 
los factores que afectan su captación. Este trabajo se estructura de la siguiente manera: primero, se presenta la metodología y equipamiento para realizar esta evaluación, luego, se propone un caso de estudio en la isla San Cristóbal (Galápagos), expuesta a la presencia de neblina y con problemas de escasez de agua para su sostenibilidad. A continuación, los resultados del caso de estudio son presentados y discutidos. Finalmente las conclusiones del trabajo son presentadas.

\section{Metodología}

\section{A. Intercepción de neblina y ubicación geográfica}

Existen básicamente tres factores que afectan la intercepción de neblina [33], [53], [54] la ubicación geográfica, diseño del colector y factores climáticos. Mientras las gotas de lluvia tienen un diámetro de 0.5 a $5 \mathrm{~mm}$, las gotas de agua en la neblina tiene un diámetro de 1 um a 40 um [32], [35]. Este pequeño tamaño es el motivo por el cual estas gotas no pueden precipitarse, y por ende flotan sobre la superficie del suelo como niebla, neblina o bruma. Del punto de vista meteorológico, la diferencia entre estos tres últimos nombres radica básicamente en el rango de visibilidad que permiten (ej. la variable climática que reduce la visibilidad a una distancia menor a $1000 \mathrm{~m}$ se considera como niebla). Para el punto práctico de este estudio, dicha diferenciación no es relevante, y por lo tanto a la niebla y bruma se los considerará como neblina. La intercepción de neblina ocurre cuando las pequeñas gotas de agua que conforman la neblina son acarreadas por el viento y chocan contra una superficie, que se satura y permite su precipitación.

La ubicación geográfica es un factor clave para la implementación de un sistema de cosecha de neblina, ya que no todas las regiones están expuestas a la presencia de neblina. Las regiones costeras serían las mayormente favorecidas por este sistema, ya que están propensas a la neblina de advección, producto de la condensación de las masas de aire caliente y cargadas de humedad, provenientes del mar, cuando pasan por el suelo frío. Este tipo de neblina puede estar presente varios cientos de kms hacia la superficie continental, es por este motivo que varias regiones áridas (ej. desierto de Ataca- 
ma, desierto del Sahara, Golfo Pérsico) están expuestas a neblina. Las regiones montañosas también son propensas a la presencia de neblina, debido a que las montañas representan un obstáculo en el movimiento de las masas de aire húmedo, obligándolas a elevarse por sus laderas y condensándose debido a enfriamiento adiabático. También, en regiones con clima templado, la neblina se puede formar debido a la condensación del aire húmedo caliente durante el día por el enfriamiento del suelo en la noche.

\section{B. Dispositivos de colección de neblina}

Existen dos alternativas para realizar la cosecha de neblina, puede ser tanto la intercepción de forma natural como de forma artificial. La intercepción de forma natural ocurre cuando el dosel de la vegetación (hojas y ramas que conforman su estructura) intercepta la neblina permitiendo el goteo del dosel hacia el suelo. En este caso, la colección se hace bajo la vegetación de forma directa. Este método se ha utilizado en sitios como en las Islas Canarias [55] y Omán [45], siendo ésta, la única fuente de agua tanto para sus habitantes como para animales [32]. Para este caso, la intercepción de la neblina va a depender de la estructura de la vegetación, de la forma de sus hojas y orientación respecto al viento. La cosecha de neblina implicaría la implementación de cisternas de recolección bajo la vegetación, lo cual puede ser complicado. También, las cisternas requerirían de un constante mantenimiento (para limpiar su superficie de ramas y hojas) y la infraestructura podría suponer una afectación al ecosistema (en el caso de bosques). La otra alternativa, es el uso de estructuras artificiales para captar neblina, como son los captadores de neblina. Los captadores tienen una malla que intercepta las pequeñas gotas de neblina a medida que el aire atraviesa la misma. Las gotas captadas en la malla se unen formando gotas más grandes que se escurren por la malla hacia una canaleta que conduce el agua a un tanque de almacenamiento.

Existen diferentes tipos de captadores de neblina, los cuales dependen de su estructura y tipo de malla. En cuanto a la estructura existen básicamente dos tipos: i) dispositivos cilíndricos (usado en Hawaii [56], Tenerife [52] y Costa Rica [57]), cuya malla constituye 
la superficie de un cilindro, y el cual está conectado a un embudo que recoge la neblina captada, y ii) dispositivos bidimensionales (usado en Chile, España, Nepal, Sudáfrica y Australia [15], [39], [45]), que consiste en una superficie plana de malla ubicada perpendicularmente a la dirección del viento.

Los dispositivos cilíndricos tienen la ventaja de no ser dependientes de la dirección del viento, la desventaja es que no se pueden construir superficies muy grandes. Los dispositivos bidimensionales se pueden construir en varios tamaños dependiendo de las necesidades, no ocupan mucho espacio, son relativamente baratos y fáciles de instalar.

Sin embargo, tienen como desventaja que cuando existen vientos muy fuertes pueden ser desestabilizados o su malla puede romperse. Existen otro tipo de colectores menos usados, como: colector macrodiamante [58], atrapaniebla DISDERA [59], tipo Juvik [56], colector Eiffel [60] y two-stage tunnel tipo Daube [57]. Otra característica importante de los colectores de neblina es su tipo de malla. Tanto el material como su diseño influyen directamente en la eficiencia del colector. Un material común para las mallas es el acero, ya sea de aluminio [56], [57] o de acero inoxidable [15] con un diseño de pantalla de persiana o entrelazada con material de poliester. Este material ha sido probado efectivo durante eventos únicamente de neblina y eventos de lluvia y neblina con vientos fuertes [57].

Otro material utilizado tanto en colectores cilíndricos como en bidimensionales es el wire harp screen, que consiste en una red de hilos de nylon con un diámetro de $0.8 \mathrm{~mm}$ espaciados $2 \mathrm{~mm}$ [57], [61]. La desventaja de este material es su pérdida de eficiencia en eventos con fuertes vientos. El material mayormente utilizado es la red de sombra Raschel [15], que es de polipropileno y cuyo diámetro de fibra es efectivo para capturar la neblina. Además, el tejido consiste en triángulos estirados que beneficia el desplazamiento vertical por gravedad de las gotas que chocan contra ella [15], [45]. Uno de los colectores más usados alrededor del mundo (alrededor de 40 países [15]), es el "standard fog collector" (SFC) o colector de neblina estándar, propuesto por Schemenauer y Cereda 1994 [45]. Este colector consiste en un panel de red de sombra Raschel con 
una superficie de recolección de $1 \mathrm{~m}^{2}$, que está sostenido por una estructura en forma de marco a 2 m de elevación perpendicular a la dirección del viento.

La elección del tipo de colector de neblina va a depender de las condiciones del sitio, facilidad de construcción y su precio. Para el caso de la evaluación de la prefactibilidad de este sistema, se recomienda utilizar al menos dos colectores de diferentes materiales. Materiales conocidos y usados en otros sitios pueden servir para realizar comparaciones sobre la capacidad de colección de neblina del sitio de estudio y hacer predicciones previas sobre el potencial de captura a largo plazo en base a estas comparaciones. En el caso de que no se conozca las condiciones climáticas previas a la instalación de los colectores (en especial la velocidad y dirección del viento), se recomienda fuertemente el uso de al menos un colector cilíndrico. Aunque la cuantificación de la captura de neblina se podría realizar a nivel diario de forma manual, sería preferible realizar mediciones automáticas a un menor paso de tiempo para realizar comparaciones con otras variables meteorológicas.

\section{Influencia de factores climáticos}

Es importante identificar los factores climáticos que afectan la neblina. De acuerdo a la literatura, los principales factores que afectan la intercepción de neblina son el contenido de agua en el aire, el tamaño de las gotas y la velocidad del viento. Los dos primeros factores tienen relación con las propiedades de la neblina, mientras la velocidad del viento es un factor climático. La dirección del viento también puede ser un factor preponderante, la elección de usar un colector cilíndrico o bidimensional depende de esta variable. La lluvia es otro factor importante, sobre todo en regiones en donde existen precipitaciones del tipo orográficas, en donde las mismas están acompañadas de neblina. Además del viento y la lluvia, la temperatura, radiación solar y humedad relativa son otras variables meteorológicas que pueden afectar a la intercepción de neblina.

La implementación de una estación meteorológica en el sitio donde se planea evaluar la intercepción de neblina es fundamental. Se debe tratar que la estación mida al menos las principales variables 
meteorológicas mientras se hace la cuantificación de la intercepción de neblina. El monitoreo de otras variables como la visibilidad y la distribución del tamaño de partículas de agua en suspensión sería un valor agregado, dado que se podría determinar el contenido de agua en el aire a partir de estos datos [62].

Para cuantificar el efecto de las variables meteorológicas en la intercepción de neblina se puede utilizar el coeficiente de correlación, $r(x, y)$. Aunque simple, el coeficiente de correlación además de reflejar la relación entre dos variables indica también el efecto multiplicador o reductor de la variable independiente sobre la dependiente. Otra forma de cuantificar este efecto es un análisis de cuantía, en donde se pueda determinar la importancia relativa de cada una de las variables climáticas en la recolección de neblina. Este análisis parte de la asunción de un modelo lineal múltiple, en donde existe una variable independiente que es función de variables independientes o predictores:

$$
y=a_{0}+a_{1} x_{1}+a_{2} x_{2}+\cdots+a_{n} x_{n}(1),
$$

donde y es la variable dependiente, $x 1,2$,..n son los predictores y $a 1,2, \ldots, n$ son los parámetros de regresión. De acuerdo a la teoría de propagación de errores, el error de una variable en función de varias viene dado por la expresión:

$$
\varepsilon(y)=\sum_{i=1}^{n}\left|\frac{\partial}{\partial x_{i}} y\right| \varepsilon\left(x_{i}\right)(2),
$$

donde $n$ es el número de predictores, $\varepsilon(y)$ y $\varepsilon\left(x_{i}\right)$ son los errores de la variable dependiente y de los predictores, respectivamente. Para el caso de medidas directas (como las variables meteorológicas), el error de los predictores puede estar descrito por su desviación estándar $\left(S x_{i}\right)$. Usando la ecuación (1) como función en la ecuación (2), y resolviendo la ecuación (2), el error de la variable dependiente viene dado por:

$$
\varepsilon(y)=a_{1} S x_{1}+a_{2} S x_{2}+\cdots+a_{n} S x_{n}(3) .
$$

Finalmente, si se normaliza el error de la variable independiente, se puede obtener la contribución relativa al error de cada una de las variables independientes $\left(P x_{i}\right)$ : 


$$
P x_{i}=\frac{a_{i} S x_{i}}{\sum_{i=1}^{n} a_{i} S x_{i}}(4)
$$

Para el caso del análisis de la intercepción de neblina, la variable dependiente corresponde a la intercepción de neblina, las variables independientes o predictores corresponden a las variables meteorológicas, y la contribución relativa al error de la variable dependiente de cada predictor se podría interpretar como el efecto relativo de cada variable meteorológica sobre la intercepción de neblina.

\section{Separación de neblina y lluvia en el colector}

Usualmente los diferentes dispositivos de colección de neblina atrapan tanto la neblina como la lluvia. En este caso no se tiene una medida del aporte real de la intercepción de neblina, una medida que es fundamental para la evaluación de la implementación de un sistema de recolección de neblina. En varios estudios [52], [56], [63], se utiliza una protección en la parte superior del colector para evitar la entrada de precipitación y medir únicamente la neblina. Sin embargo, los objetivos de estos estudios es la medición únicamente de la neblina y no el estudio de la factibilidad de un sistema de colección de neblina como es el caso de esta investigación.

La cuantificación de neblina es necesaria, por lo que una alternativa sería que además de los colectores de neblina se instale un dispositivo para realizar la medición únicamente de la neblina. Se debería considerar que el uso de un protector de lluvia en el dispositivo de colección de neblina, podría causar un efecto aerodinámico, alterando la dirección e intensidad del viento y por ende, afectando la estimación de neblina. Otra alternativa es realizar una separación de los aportes de neblina y lluvia en los colectores. Esta separación resulta ser una medida más directa del aporte de neblina ya que se la realiza directamente sobre el colector que se estaría evaluando y no indirectamente de otro dispositivo en donde se mide únicamente la neblina.

Para realizar la separación de neblina se puede utilizar un modelo geométrico sencillo. Domínguez et al. (2017) [43] utilizó este modelo para separar la neblina de la lluvia en un colector cilíndrico, al determinar primero el valor del volumen real de precipita- 
ción captado por el colector de neblina, y calculando el volumen de neblina, como la diferencia entre el volumen medido por el colector y el volumen real de la lluvia (Figura 1).

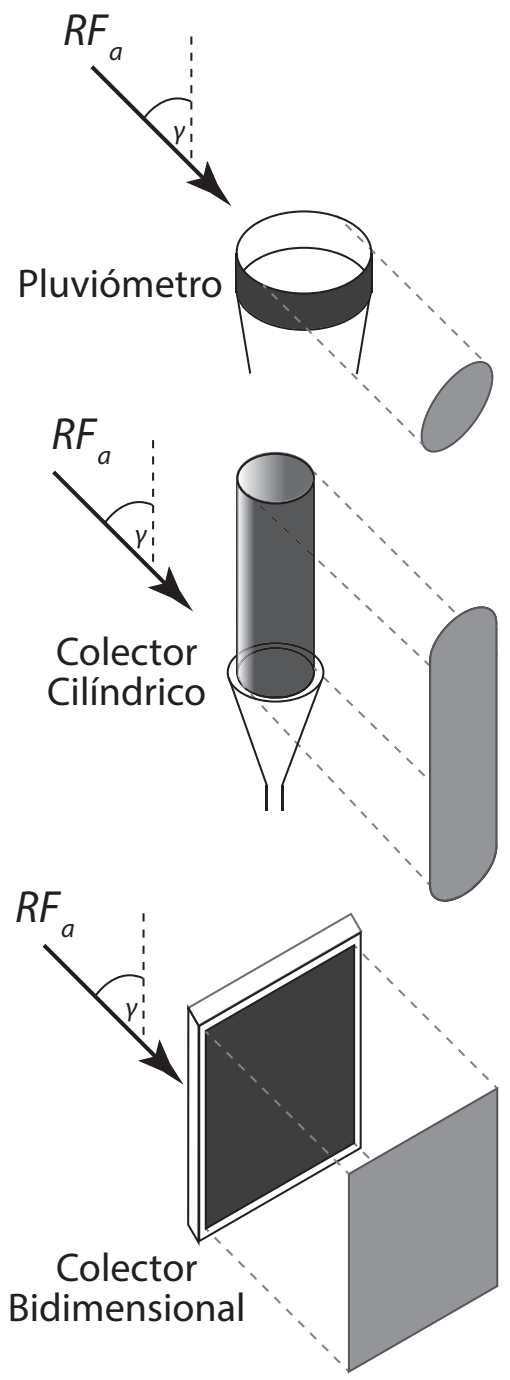

Figura 1. Área proyectada de un pluviómetro, colector cilíndrico y un colector bidimensional, perpendicular a la dirección de la precipitación 
Esta metodología se basa en que la intensidad y dirección de la lluvia captada por un pluviómetro normal y el colector de neblina es la misma en ambos dispositivos. Esto puede ocurrir, si ambos dispositivos están ubicados cerca el uno del otro. También, es necesario asumir que la intercepción de neblina se produce sobre la proyección perpendicular a la dirección del viento de la malla del colector. En este caso, se necesitaría un medidor de velocidad y dirección del viento tridimensional. En el caso de tener un medidor estándar de la dirección del viento (únicamente mide la dirección horizontal del viento), la componente vertical de la dirección se podría asumir igual a la pendiente del terreno o de forma horizontal. Para casos de topografía sencilla y pendientes suaves se podría asumir una componente horizontal del viento. Pero en casos de topografía complicada o de pendientes fuertes se debería optar por la medición tridimensional o el uso de la pendiente del terreno, respectivamente. El modelo se describe a continuación.

Ante la existencia de viento (condición necesaria para que exista intercepción de neblina), el pluviómetro mide la componente vertical de la lluvia $\left(R F_{v}\right)$ que viene dado de una relación trigonométrica:

$$
R F_{v}=R F_{a} \times \cos \gamma(5),
$$

donde $\left(R F_{a}\right)$ es la magnitud de la lluvia real que tiene una inclinación (angulo). El ángulo de inclinación de la lluvia se calcula a partir de la intensidad de la precipitación, el tamaño y velocidad terminal de caída de las gotas de lluvia, y de la velocidad del viento [40], [43]. La intensidad de la lluvia corresponde a la medición del pluviómetro directamente. El tamaño promedio del diámetro de una gota de lluvia (D) en mm puede ser calcula con [40], [43]:

$$
R I=2.23 \times\left(0.03937 \times R F_{v}\right)^{0.102}(6),
$$

donde $R I$ es la intensidad de la lluvia en $\mathrm{mm} / \mathrm{h}$. La velocidad de caída de la gota $(U)$ viene dado directamente de su diámetro [40], [43], [64]:

$$
V=3.378 \times \ln (R I)+4.213(7)
$$

y el ángulo de caída se calcula con [40], [43], [65]: 


$$
\tan \gamma=\frac{U}{V}(8)
$$

donde $V$ es la velocidad del viento.

A partir de la ecuación (5) se puede determinar la magnitud de la lluvia real, la cual es equivalente al volumen de lluvia real dividido para la proyección de la superficie circular horizontal del pluviómetro perpendicular a la dirección de $R F_{a}$. Realizando la misma equivalencia, el volumen captado por el colector de neblina correspondiente a la lluvia real $\left.(V R)_{a}\right)$ es [43]:

$$
V R_{a}=R F_{a} \times S_{r}(9),
$$

donde $S_{r}$ es la proyección de la superficie del colector perpendicular a la dirección de $R F_{a}$. Finalmente, la magnitud de la intercepción de neblina captada por el colector $(N I)$ está dada por [43]:

$$
N I=\frac{\left(V F_{t}-f e \times V R_{a}\right)}{S_{v}}(10),
$$

donde $S_{v}$ es la proyección de la superficie del colector perpendicular a la dirección del viento y es un coeficiente de corrección aplicado a la medición de la lluvia en el colector [43].

La dificultad de este método resulta en la obtención de las superficies proyectadas del colector de neblina, ya que va a depender de la dirección del viento y del ángulo de caída de la precipitación para cada paso de tiempo. En el caso de un colector bidimensional o cilíndrico, esta estimación no resulta difícil debido a que su geometría es relativamente sencilla, pero en el caso de geometrías complicadas (caso del colector tridimensional o colector con secciones complementarias) la estimación de esta superficie puede ser bastante desafiante.

\section{Caso de estudio (San Cristóbal, Galápagos)}

\section{A. Área de estudio}

\section{1) Ubicación geográfica y clima}

El Archipiélago de Galápagos está ubicado a 1000 km al oeste del Ecuador continental. La isla San Cristóbal se ubica al lado este del Archipiélago. La isla tiene una superficie de $558 \mathrm{~km}^{2}$, donde más del 80\% de su área está protegida por el Parque Nacional Galápagos. 
La población total de la isla es de aproximadamente 7500 habitantes [66], donde la mayor concentración de la población está en Puerto Baquerizo Moreno. Las condiciones climáticas de Galápagos están influenciadas por la interacción de la corriente oceánica de Humboldt y los vientos alisios [67], [68]. Los vientos alisios, con dirección Sudeste, se condensan en las pendientes del barlovento de las islas principales generando una diferencia clara entre el lado húmedo barlovento y el lado seco sotavento de las islas. La migración interanual de la zona de convergencia intertropical da lugar a la aparición de dos temporadas climáticas, la fría de "garúa" y la cálida de "invierno". La temporada de garúa, ocurre entre junio y diciembre, y se caracteriza por la presencia de temperaturas relativamente bajas tanto del aire como de la superficie del océano [68]. Las precipitaciones son del tipo orográficas, de larga duración, baja intensidad y localizadas en el lado barlovento de las islas [68]. Durante esta época, una capa de inversión térmica se forma en la parte alta de las islas, dificultando el paso de las masas de aire húmedo y conduciendo de esta manera a la formación de neblina en las partes altas de las islas [69] La presencia de neblina es casi permanente durante esta época. Por otra parte, la temporada de invierno, ocurre de enero a mayo, y se caracteriza por la existencia de temperaturas relativamente altas en el aire y la superficie del océano. En esta época, ocurren precipitaciones del tipo convectivo, de corta duración y alta intensidad [68], [70], [71].

\section{2) Geología y recursos hídricos}

Las islas Galápagos, formadas de rocas basálticas mixtas [72][75], emergen de un punto caliente en una plataforma submarina poco profunda que forma la parte occidental de la cordillera Carnegie Ridge, en la placa de Nazca [75]. Pero la presencia de una cresta segmentada en el océano Centro de Propagación de Galápagos (GSC) no se puede ignorar, ya que afecta la estructura y organización de las islas. La isla San Cristóbal está formada por la erupción de flujos de lava pahoehoe y aa' [72] intercalado por periodos de calma, en donde debido a la condiciones húmedas en el clima, capas de suelo se desarrollaron debido a la meteorización [75]. Por este motivo, la estructura interna de la isla posee un sistema multicapas entre flujos de lava y paleo-suelos. Al final de la actividad volcánica, la isla fue cubierta 
por una capa de depósitos de piroclastos de al menos $10 \mathrm{~m}$ [72], que debido a la meteorización se formó un suelo en la superficie.

En el lado barlovento de la isla, las condiciones húmedas y la presencia de suelo favorecieron la escorrentía promoviendo la erosión. Por este motivo se puede observar una red de drenaje desarrollado que tiene incisiones profundas en varios sectores. La hidrología es compleja en este lado de la isla, en la parte alta existen praderas con pendientes suaves que favorecen el encharcamiento de agua, como es el caso de la laguna "El Junco". A media elevación, se puede observar varias encañadas. Algunas secas y otras con agua permanente que nacen de fuentes que están siendo alimentadas por un acuífero colgado formado sobre una capa de baja permeabilidad que no permite la percolación del agua infiltrada a fondo [74]-[77]. El caudal de la mayoría de encañadas es menor a 5 1/s [78]. En la parte costera, el agua de las encañadas se vuelve a reinfiltrar, y solamente cuatro encañadas llegan a descargarse en el océano en la bahía de agua dulce [79].

Galápagos se caracterizan por la falta de agua en sus islas. La única isla con fuentes de agua dulce permanente es la isla San Cristóbal. El suministro de agua para la población es captado de dos encañadas, "La Toma" y "Cerro Gato", proporcionando alrededor de 30 l/s para la población de Puerto Baquerizo Moreno. Aunque actualmente las necesidades de agua para uso doméstico están satisfechas (aparentemente), existe un déficit importante en cuanto al riego. En el 2010, existió un déficit de 20.5\% equivalente a $1.8 \mathrm{hm}^{3}$ [80], y se estima que este déficit se incremente a $7.1 \mathrm{hm}^{3}$ (80.1\%) para el 2035 [81]. Este problema es aún mayor en otras islas poblados donde no existe fuentes de agua dulce permanente. Para garantizar las sostenibilidad de las poblaciones en estas islas, es necesario encontrar fuentes alternativas de agua, como es el caso de la cosecha de neblina.

\section{B. Implementación de la metodología}

\section{1) Sitio de estudio}

El sitio de estudio está ubicado en la parte alta del lado sur barlovento de la isla San Cristóbal, a 600 m.s.n.m (Figura 2). 

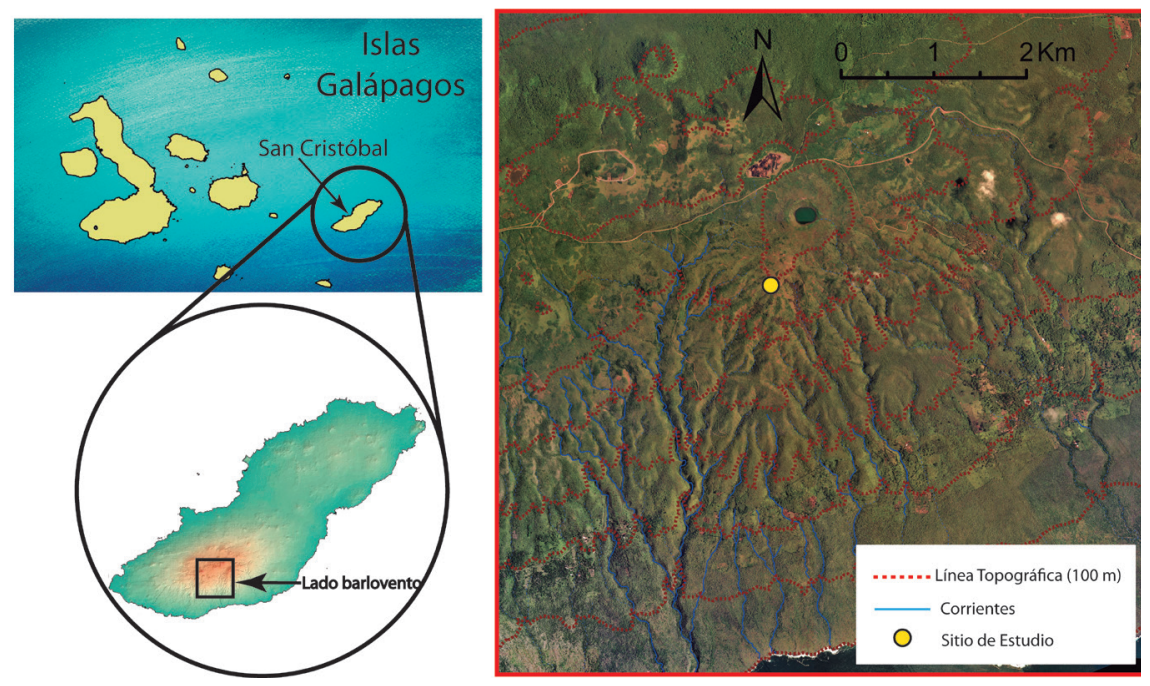

Figura 2. Ubicación del sitio de estudio en el lado barlovento de la Isla San Cristóbal (Galápagos)

Este sitio está dentro del área agrícola de la isla que se extiende desde la parte a media elevación de la isla (aproximadamente a 200 m.s.n.m) hasta la cumbre de la misma. En los alrededores del sitio, el suelo está cubierto por pasto y por bosques de Miconia Robinsoniana, una especie endémica de Galápagos. Toda esta zona (desde 300 m.s.n.m) está expuesta a la presencia de neblina alrededor de seis meses al año durante la época de garúa, por lo que representa un sitio ideal para evaluar y cuantificar la intercepción de neblina.

\section{2) Monitoreo climático}

Para realizar el monitoreo de las principales variables meteorológicas se utilizó una estación climática (Tabla 1).

Tabla 1. Equipos de la estación climática a 600 m.s.n.m. en San Cristóbal (Galápagos)

\begin{tabular}{|c|c|c|}
\hline Equipo & Marca & Modelo \\
\hline Datalogger $^{\mathrm{a}}(\mathrm{x} 2)$ & Campbell & CR1000 \\
\hline Pluviómetro $^{\mathrm{b}}$ & Texas & TE525MM \\
\hline Pluviómetro $^{\mathrm{c}}(\mathrm{x} 2)$ & Précis Mécanique & R307A0 \\
\hline
\end{tabular}




\begin{tabular}{|c|c|c|}
\hline Equipo & Marca & Modelo \\
\hline Pluviómetro $^{\text {d }}$ & Campbell & ARG100 \\
\hline $\begin{array}{c}\text { Sensor de temperatura y hume- } \\
\text { dad relativa }\end{array}$ & Campbell & CS215 \\
\hline Anemómetro & Young & $03002-5$ \\
\hline Piranómetro & Campbell & CS300 \\
\hline
\end{tabular}

a. Registrador de datos.

b. Usado para la medición de la precipitación.

c. Pluviómetro modificado para la medición de la intercepción de neblina en los colectores estándar (SFC35 y SFC50).

d. Pluviómetro modificado para la medición de la intercepción de neblina en el colector cilíndrico (CFC).

La precipitación se midió con un pluviómetro Texas TE525MM ubicado a $1.5 \mathrm{~m}$ del suelo. La dirección y velocidad del viento se monitoreó a 2 m sobre el nivel del suelo con un sensor Young WindSentry Kit. La radición solar se midió con un piranómetro de silicona Kipp\&Zonen SPLite a $2 \mathrm{~m}$ del suelo. La humedad relativa y temperatura se monitorearon con un sensor Campbell CS215 a $2 \mathrm{~m}$ sobre el suelo. Todos los datos de estas variables se grabaron en un datalogger Campbell CR1000, que permite realizar mediciones cada minuto y registra los datos cada 15 minutos.

La intercepción de neblina se midió utilizando tres colectores (Figura 3).

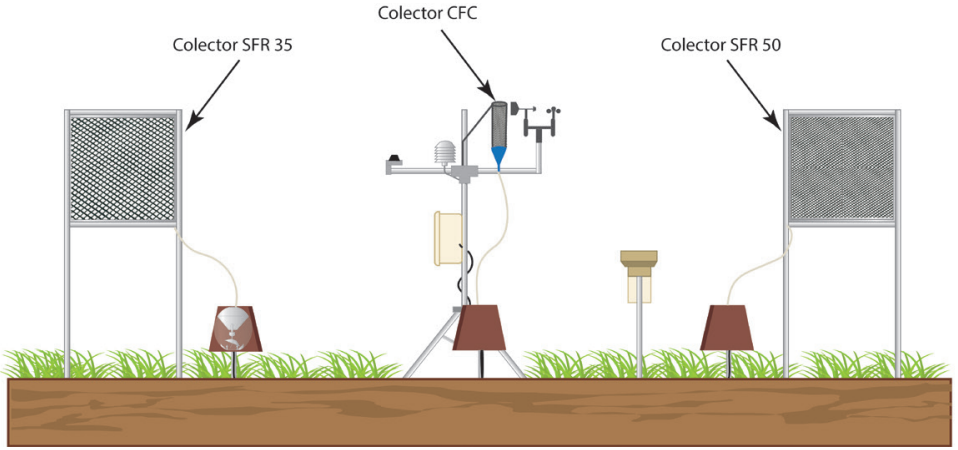

Figura 3. Esquema de monitoreo meteorológico y de intercepción de neblina. La estación meteorológica cuenta con un pluviómetro (1.5 m sobre el suelo), un anemómetro ( $2 \mathrm{~m}$ sobre el suelo), un sensor de temperatura y humedad relativa ( $2 \mathrm{~m}$ sobre el suelo), un piranómetro ( $2 \mathrm{~m}$ sobre el suelo) y tres colectores de neblina conectados a basculadores automáticos 
El primero, es un colector cilíndrico ( $\mathrm{CFC}$ ), con una altura de $40 \mathrm{~cm}$ y $12 \mathrm{~cm}$ de diámetro, que tiene una estructura metálica cubierta por una malla plástica de $1 \mathrm{~mm}$ de apertura y un coeficiente de sombra del 72\% (estimado a partir de análisis de imágenes). El agua interceptada por la malla se drena en un embudo en la parte inferior del colector, el cual está conectado a una manguera que dirige el agua hacia un sistema de basculación automático, el cual permite la medición del volumen de agua interceptado por el colector. Los otros dos dispositivos de medición de neblina, fueron los conocidos colectores estándar de neblina ubicados a $2 \mathrm{~m}$ sobre el nivel del suelo, con una superficie de colección de $1 \mathrm{x} 1 \mathrm{~m}$, y que tienen el mismo diseño pero difieren en la malla utilizada. Los colectores fueron construidos con malla de polietileno, de $35 \%$ de coeficiente de sombra en el primero (SFC35), mientras que el segundo colector tiene una malla de $50 \%$ de coeficiente de sombra (SFC50). La estructura de soporte de colector fue construida con tuberías de PVC, que resultó ser un material relativamente barato, de fácil transporte y que no afectó la sensibilidad del ecosistema de Galápagos. Estos colectores tienen una canaleta de recolección conectada a una manguera que dirige el agua captada por los colectores a sistemas de basculación automáticos individualmente. Los datos de los tres colectores fueron grabados por otro datalogger CR1000 que registra los datos de forma simultánea con los datos del resto de variables meteorológicas.

\section{3) Aplicación de la metodología}

La evaluación de la intercepción de neblina se realizó en los meses de junio y julio del año 2016, al inicio de la temporada de garúa. La cuantificación del rendimiento de los colectores para captar agua se lo realizó de dos maneras: i) con el volumen acumulado directamente medido por los colectores en $1 / \mathrm{m} 2 \cdot$ día, y ii) utilizando el modelo geométrico de separación entre intercepción de neblina y la lluvia a nivel horario, en $\mathrm{mm}$. Para poder utilizar el modelo, se estimó el área proyectada de los colectores en el plano perpendicular a la dirección de la lluvia a nivel horario, en $\mathrm{mm}$. Para poder utilizar el modelo, se estimó el área proyectada de los colectores en el plano perpendicular a la dirección de la lluvia (S_r) para cada paso de tiempo. En el caso del colector cilíndrico, S_r se 
calculó en la forma descrita en Dominguez et al., 2017 [43], y para el caso de los SFCs, se utilizaron varias relaciones geométricas. Para todos los dispositivos, se asumió que el área de intercepción de proyección perpendicular a la dirección del viento es perpendicular a la pendiente del terreno. $S_{v}$ fue estimado con las mismas relaciones trigonométricas utilizadas para obtener $S_{r}$, y es un valor constante para todo el periodo de estudio. El factor fe, correspondiente para la corrección de medición de la precipitación se estimó usando la metodología descrita en Domínguez et al., 2017 [43].

El análisis estadístico se realizó utilizando un filtro, primero los registros se discretizaron a datos horarios, usando la media o sumatoria de los datos cada 15 minutos. Luego, se utilizaron únicamente los datos en que existió un registro de intercepción de neblina diferente de 0 , de esta manera se evalúa únicamente la relación entre las variables meteorológicas con la intercepción de neblina, cuando ésta ocurre.

\section{Resultados}

\section{A. Condiciones climáticas durante el periodo de estudio}

A partir de los datos históricos de la estación operada por la Fundación Charles Darwin en la costa de Santa Cruz, se puede observar que durante el periodo de estudio (junio y julio de 2016), la precipitación acumulada es de $26.5 \mathrm{~mm}$ y la temperatura media del aire es de $23.3{ }^{\circ} \mathrm{C}$, que comparado, con los valores promedio de la estación (44.7 $\mathrm{mm}$ para la precipitación y $23.3{ }^{\circ} \mathrm{C}$ para la temperatura), resultan condiciones secas en términos de precipitación y condiciones normales en términos de temperatura.

Durante los meses de junio y julio de 2016 en el sitio de estudio la estación meteorológica registró las principales variables meteorológicas. Un resumen de esta información se presenta en la Tabla 2.

Tabla 2. Resumen de los valores diarios de las principales variables meteorológicas registras en el sitio de estudio

\begin{tabular}{|c|c|}
\hline Variable & Valor \\
\hline Precipitación $^{\mathrm{a}}[\mathrm{mm}]$ & $72.0(23.9)$ \\
\hline Temperatura $^{\mathrm{b}}\left[{ }^{\mathrm{o}} \mathrm{C}\right]$ & $18.2(15.5 \mathrm{a} 24.0)$ \\
\hline
\end{tabular}




\begin{tabular}{|c|c|}
\hline Variable & Valor \\
\hline${\text { Radiación } \operatorname{solar}^{\mathrm{c}}\left[\mathrm{W} / \mathrm{m}^{2}\right]}$ & $211(56$ a 318) \\
\hline Humedad relativa $^{\mathrm{d}}[\%]$ & $99(67)$ \\
\hline Velocidad del viento $^{\mathrm{e}}[\mathrm{m} / \mathrm{s}]$ & $2.5(1.9$ a 3.1) \\
\hline Dirección del viento $^{\mathrm{e}}\left[{ }^{\mathrm{O}}\right]$ & $199(187$ a 217) \\
\hline
\end{tabular}

a. Precipitación acumulada en todo el período corregida en función del ángulo de caída. En paréntesis, la precipitación máxima diaria registrada.

b. Temperatura media diaria. En paréntesis, el valor mínimo y máximo registrado.

c. Radiación solar media diaria durante las horas de sol. En paréntesis, el valor del primer y tercer cuartil.

d. Humedad relativa media diaria. En paréntesis, el porcentaje del tiempo que la humedad relativa fue mayor o igual a $99 \%$.

e. Variable media diaria. En paréntesis, el valor del primer y tercer cuartil de la variable.

La precipitación registrada alcanzó un valor de $72 \mathrm{~mm}$ y la temperatura media fue de $18.2^{\circ} \mathrm{C}$. Estos valores resultan bajos comparando con los registros Junio y Julio del 2013 al 2016 en el mismo sitio (286.2 $\mathrm{mm}$ y $19.1^{\circ} \mathrm{C}$ para la precipitación y temperatura, respectivamente). La velocidad y dirección media y diaria del viento fue de $2.5 \mathrm{~m} / \mathrm{s}$ y $199^{\circ}$, respectivamente. La dirección del viento es similar a años pasados $\left(193^{\circ}\right)$, pero en el caso de la velocidad del viento, este valor está por debajo de los registrados en años pasados $(2.9 \mathrm{~m} / \mathrm{s})$. La radiación solar media diaria durante las horas de sol permaneció relativamente baja durante todo el periodo $\left(211 \mathrm{~W} / \mathrm{m}^{2}\right)$ y es ligeramente mayor que la presentada en años pasados $\left(202 \mathrm{~W} / \mathrm{m}^{2}\right)$. Similar a años pasados, la humedad relativa estuvo cerca o igual a condiciones de saturación. La humedad relativa estuvo por encima del $95 \%$ en todo el periodo y durante el $67 \%$ del tiempo, ésta fue mayor al $99 \%$.

\section{B. Cuantificación de la intercepción de neblina y comparación entre colectores}

Durante el periodo de estudio, el volumen total colectado por el colector de neblina CFC, SFC35 Y SFC50 fue de 26, 337 y 410 l, respectivamente. Estos valores representan un rendimiento diario de $3.8 \mathrm{l} / \mathrm{m}^{2} \cdot$ día para el colector $\mathrm{CFC}, 7.0 \mathrm{l} / \mathrm{m}^{2}$.día para el colector SFC35 y $8.5 \mathrm{l} / \mathrm{m}^{2}$.día para el colector SFC50 (Tabla 3 ). 


\section{Tabla 3. Rendimiento e intensidad de intercepción de neblina de los tres colectores estudiados}

\begin{tabular}{|c|c|c|c|}
\hline Variable & CFC & SFC35 & SFC50 \\
\hline Rendimiento $^{\mathrm{a}}\left[1 / \mathrm{m}^{2} \bullet\right.$ día $]$ & $3.8(0.6 \mathrm{a} 4.9)$ & $7.0(2.0 \mathrm{a} 10.0)$ & $8.5(2.2 \mathrm{a} 12.1)$ \\
\hline Intensidad $^{\mathrm{b}}[\mathrm{mm} /$ día $]$ & $3.7(1.2 \mathrm{a} 4.6)$ & $4.1(2.0 \mathrm{a} 5.2)$ & $4.8(2.2 \mathrm{a} 5.5)$ \\
\hline
\end{tabular}

a. Volumen de neblina y precipitación captada por el colector.

b. Altura de neblina captada por el colector como resultado del modelo geométrico de separación entre neblina y precipitación.

Los valores presentados entre paréntesis corresponden al primer y tercer cuartil de la variable.

El mayor volumen colectado a un nivel diario se registró en el colector SFC50, con un valor de $44.6 \mathrm{l} / \mathrm{m}^{2}$ día. En todos los casos se logró colectar al menos $1 \mathrm{l} / \mathrm{m}^{2}$.día durante el 77\% del tiempo, y en el colector SFC50 durante el 88\% del tiempo.

Se usó el modelo geométrico descrito anteriormente para separar la neblina de la precipitación. Los resultados del modelo muestran que la altura de intercepción de neblina durante el periodo de estudio fue de $176.5 \mathrm{~mm}, 195.8 \mathrm{~mm}$ y $230.6 \mathrm{~mm}$ en el colector CFC, SFC35 y SFC50, respectivamente. Estos valores representan una intensidad de intercepción de neblina media de $3.7 \mathrm{~mm} /$ día en el colector CFC, $4.1 \mathrm{~mm} /$ día en el colector SFC35 y $4.8 \mathrm{~mm}$ /día en el colector SFC50. La altura de intercepción de neblina presentó un alto porcentaje respecto a la precipitación, alcanzando el 245\%, el 272\% y el $320 \%$ de la precipitación en el colector CFC, SFC35 y SFC50, respectivamente.

Analizando los valores de intercepción de neblina a nivel diario, se realizó una correlación entre los volúmenes captados por los colectores, y también entre la altura de agua captado por los colectores (resultados del modelo). La correlación entre los tres colectores, tanto para el volumen como para la altura, es alta en todos los casos (Tabla 4).

\section{Tabla 4. Correlación entre los colectores}

\begin{tabular}{|c|c|c|c|}
\hline Variable & CFC & SFC35 & SFC50 \\
\hline CFC & 1 & 0.87 & 0.95 \\
\hline SFC35 & 0.70 & 1 & 0.97 \\
\hline SFC50 & 0.76 & 0.96 & 1 \\
\hline
\end{tabular}

Los valores con fondo blanco y gris representan el factor de correlación del volumen de neblina y precipitación captada entre colectores y la altura de neblina captada (resultado del modelo geométrico) entre colectores, respectivamente. 
Los colectores estándar (SFC) presentan una mayor correlación entre sí que la que se presenta con el colector cilíndrico, sobre todo en el caso de la altura de neblina.

\section{Influencia de las variables meteorológicas en la intercepción de neblina}

Se realizó un análisis sobre la influencia de las variables meteorológicas en la intercepción de neblina de los colectores. En la Tabla 5 se muestra la correlación existente entre las variables meteorológicas y la intercepción de neblina en cada colector. La correlación es pobre para todos los casos. La variable que presenta mayor correlación con los tres colectores es la velocidad del viento (0.47 a 0.55). En el caso del colector CFC, la correlación con la precipitación también presenta un valor relativamente alto. En el caso de los colectores SCF, la otra variable que presenta una correlación relativamente alta es la radiación solar, aunque en este caso las correlaciones son negativas mostrando que esta variable tendría un efecto reductor en la intercepción de neblina. Para estos colectores (SFC), la correlación con la precipitación también es negativa, aunque la correlación es baja (-0.04 a -0.01).

Tabla 5. Correlación entre las variables meteorológicas y la intercepción de neblina en los colectores

\begin{tabular}{|c|c|c|c|}
\hline Variable meteorológica & CFC & SFC35 & SFC50 \\
\hline Precipitación & 0.24 & -0.04 & -0.01 \\
\hline Velocidad del viento & 0.45 & 0.47 & 0.55 \\
\hline Dirección del viento & 0.09 & 0.09 & 0.09 \\
\hline Humedad relativa & 0.05 & 0.11 & 0.04 \\
\hline Temperatura & 0.07 & 0.05 & 0.16 \\
\hline Radiación solar $^{\mathrm{a}}$ & $0.05(-0.03)$ & $-0.18(-0.29)$ & $-0.07(-0.19)$ \\
\hline
\end{tabular}

a. El valor entre paréntesis corresponde a la correlación entre la radiación solar y la intercepción de neblina únicamente durante las horas de sol

El análisis de cuantía se utilizó para determinar la influencia relativa de cada variable meteorológica en la variabilidad de la intercepción de neblina. Los resultados se muestran en la Tabla 6. 
Tabla 6. Contribución relativa de las variables meteorológicas en la variabilidad de la intercepción de neblina en los colectores

\begin{tabular}{|c|c|c|c|}
\hline Variable meteorológica & CFC & SFC35 & SFC50 \\
\hline Precipitación & 22.6 & 8.5 & 11.6 \\
\hline Velocidad del viento & 54.2 & 49.1 & 54.5 \\
\hline Dirección del viento & 5.2 & 1.1 & 2.8 \\
\hline Humedad relativa & 5.0 & 3.1 & 1.1 \\
\hline Temperatura & 2.3 & 4.8 & 7.1 \\
\hline Radiación solar & 10.7 & 33.4 & 22.9 \\
\hline
\end{tabular}

Los valores de la contribución de cada variable en la intercepción de neblina se presentan como porcentaje.

Las variables que mayor influencian tienen en la intercepción de neblina son la velocidad del viento, radiación solar y la precipitación. La velocidad del viento es la variable que mayor influencia tiene en la intercepción de neblina de los tres colectores, con una contribución aproximada del 50\%. En los colectores estándar, se puede apreciar que la radiación solar tiene una contribución importante, sobre todo en el colector SFC35, alcanzando un valor del 33\%. En estos colectores, la precipitación podría ser considerados como un factor influyente en menor medida, alcanzando un valor del aproximado del 10\%. A diferencia de los colectores estándar, en el colector cilíndrico la intercepción de neblina está mayormente influenciado por la precipitación (22.6\%) que por la radiación solar (10.7\%).

\section{Discusión}

\section{A. Influencia del clima}

El análisis de cuantía determinó la influencia de las principales variables meteorológicas. La velocidad del viento se consolidó como la variable que mayor influencia tiene sobre la intercepción de neblina, alcanzando el $54.2 \%, 49.1 \%$ y el 54 . 5\% en el colector CFC, SFC35 y SFC50, respectivamente. Estos resultados coinciden con otros estudios en donde se asevera que la velocidad del viento es la variable más influyente en la recolección de agua de neblina [54], [82] (Ritter et al., 2008; Schemenauer \& Joe, 1989). Aunque la correlación directa entre la velocidad del viento y la intercepción 
de neblina en los colectores no es buena $(<0.55)$, la influencia puede ser evidenciada con un gráfico de caja-bigote (Figura 4).

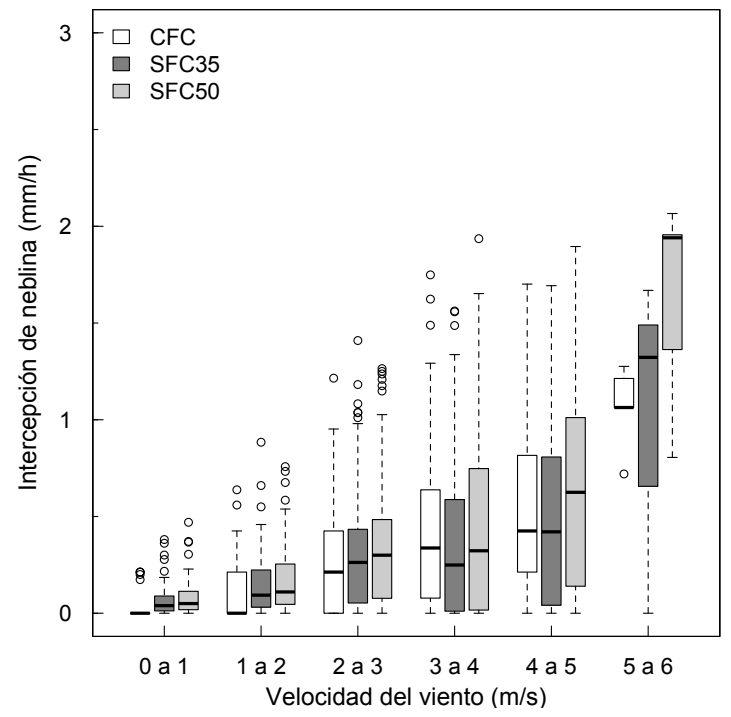

Figura 4. Estadísticas de la intensidad de intercepción de neblina en cada colector clasificadas por intervalos de velocidad del viento durante el periodo de estudio (Junio-Julio 2016)

En los tres colectores, es evidente que la intercepción de neblina incrementa conforme la velocidad del viento incrementa, sobre todo en los casos de velocidades del viento altas (mayores a $3 \mathrm{~m} / \mathrm{s}$ ). En todos los rangos de velocidad, se observa que existió intercepción de neblina en los tres colectores. La intercepción de neblina puede ocurrir por dos procesos principales, deposición e impactación [83]. En condiciones de velocidad del viento baja, la intercepción de neblina es controlada por deposición principalmente, por lo que es necesario que el contenido de agua en la neblina sea relativamente alto y que la superficie de intercepción sea eficiente. Con velocidad del viento alta, la impactación es el principal mecanismo de intercepción de neblina dado que el contenido de agua en la neblina es inversamente proporcional a la velocidad del viento debido al incremento de la evaporación potencial [83]. Esto quiere decir que superado cierto 
valor de velocidad del viento el contenido de agua en la neblina se va a reducir al punto en que la intercepción de neblina se reduzca. De hecho, Villegas et al., 2008 [83] concluye que las condiciones más óptimas de intercepción de neblina son cuando existe un contenido de agua en la neblina promedio y velocidades del viento promedio. En este caso de estudio, se puede apreciar que el contenido de agua en la neblina es relativamente alto, dado que no se alcanzó el umbral de velocidad del viento en donde la intercepción de neblina se reduzca. Se podría deducir que este hecho evidencia el potencial de intercepción de neblina en la parte alta de San Cristóbal, donde incluso con una reducción en el contenido de agua en la neblina (efecto que podría presentarse debido al cambio climático) todavía se tendría condiciones óptimas de intercepción de neblina.

La dirección del viento no tuvo una influencia importante en la intercepción de neblina de los colectores, incluyendo los bidimensionales (SFC35 y SFC50). Esto puede ser explicado porque la dirección del viento se mantuvo relativamente constante durante todo el periodo (Figura 5).

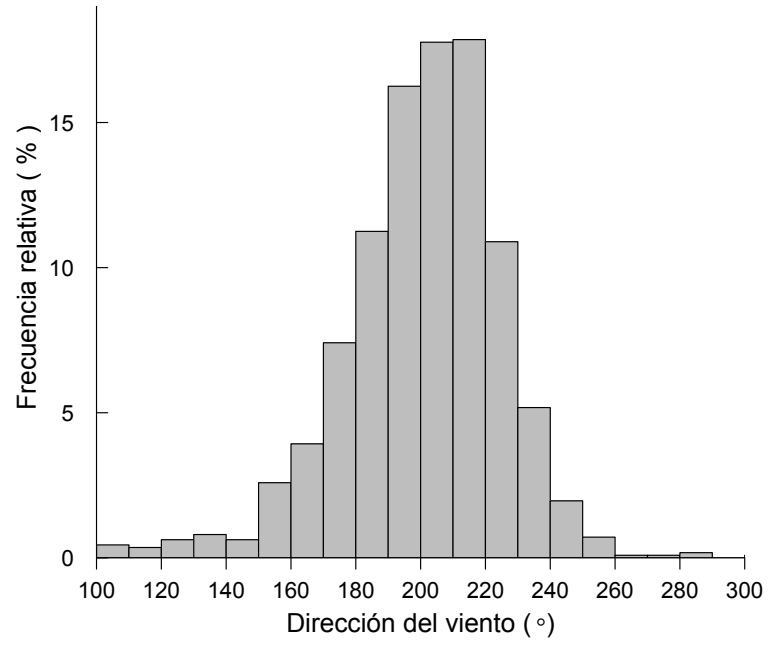

Figura 5. Diagrama de frecuencias relativas de la dirección del viento en todo el período de estudio, Junio-Julio 2016 
Durante el 90\% del tiempo en que se registró intercepción de neblina en alguno de los colectores, la dirección del viento se mantuvo entre 170 y $230^{\circ}$, que correspondería apenas en una reducción máxima del 13\% de la intercepción de neblina en la dirección perpendicular al área de colección de los colectores. Estas condiciones son ideales para el uso de un colector bidimensional sobre un cilíndrico, siendo el primero más sencillo de construir, ocupa menos espacio para una misma área de colección y resulta más barato (en la siguiente sección se discutirá su eficiencia).

La radiación solar también presentó una influencia importante en la intercepción de la neblina en todos los colectores. Su contribución en la intercepción de neblina es la más variable alcanzando un valor de $10.7 \%$ en el colector CFC, 33.4\% en el colector SFC35 y $22.9 \%$ en el colector SCF50. Existe una influencia apreciable entre la magnitud de la radiación solar durante las horas de sol y la intensidad de la intercepción de neblina por los colectores (Figura 6), siendo la intercepción de neblina por los colectores mayor cuando la radiación solar es más baja. Por esto, la radiación solar puede ser una variable que evidencie la presencia de neblina y de su contenido de agua en el aire.

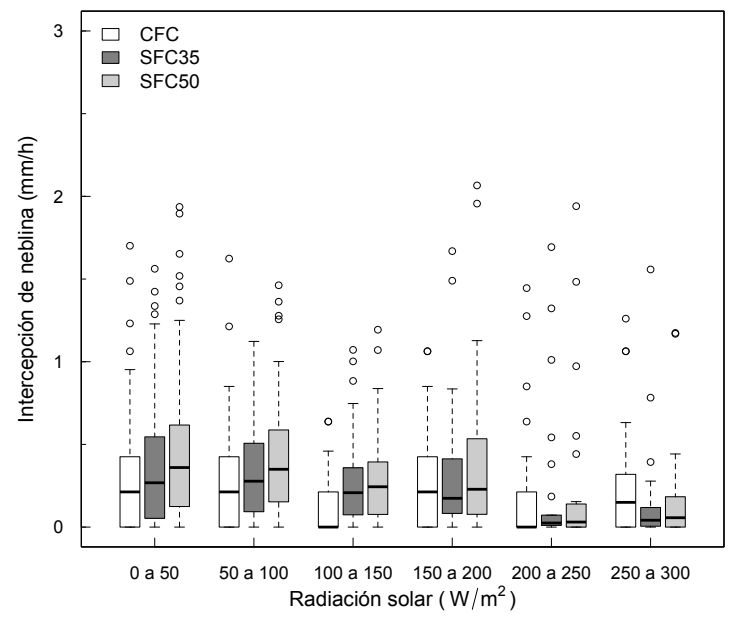

Figura 6. Estadísticas de la intensidad de intercepción de neblina en cada colector clasificadas por intervalos de radiación solar durante el periodo de estudio (junio-julio 2016) 
La intercepción de neblina durante las horas del día ocurrió durante el 38\%, el 39\% y el 41\% del tiempo en el colector CFC, SFC35 y SFC50, respectivamente. Esto quiere decir que durante mayor tiempo (aproximadamente el 60\%), la intercepción de neblina ocurrió en la noche. La radiación solar promedio cuando existió intercepción de neblina en alguno de los colectores fue de $133 \mathrm{~W} / \mathrm{m}^{2}$. Este valor resulta bajo comparado con la radiación solar promedio cuando no existió intercepción de neblina $\left(285 \mathrm{~W} / \mathrm{m}^{2}\right)$ y peor con la radiación solar promedio para cielo despejado $\left(441 \mathrm{~W} / \mathrm{m}^{2}\right)$ calculado a partir de la radiación extraterrestre. Sin embargo, esto puede ser debido a que la intercepción de neblina pudo ocurrir en horarios muy tempranos o muy tarde del día. Esto no es correcto ya que la intercepción de neblina ocurrió durante todo el día (Figura 7), presentándose las mayores magnitudes de intercepción de neblina en los colectores alrededor de las 7 de la mañana y las menores magnitudes alrededor de las 1 de la tarde. La reducción de la radiación solar debido a la presencia de neblina ya se ha evidenciado en otros sitios, como es el caso del lado sotavento de Hawaii, en el cual la neblina redujo en 36\% la insolación, en Puerto Rico el 48\% y en las Islas Canarias el 58\% [54], [84], [85]. En el caso de San Cristóbal, la reducción de la insolación fue relativamente baja alcanzando un valor del $30 \%$.

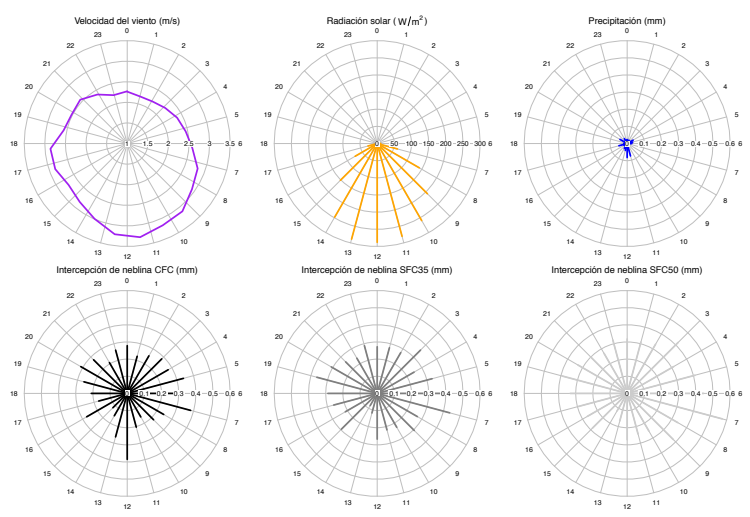

Figura 7. Variación horaria de las variables meteorológicas más influyentes en la intercepción de neblina (velocidad del viento, radiación solar y precipitación) e intercepción de neblina por cada colector durante el periodo de estudio (Junio-Julio 2016) 
La precipitación es otra de las variables que afecta la intercepción de neblina pero en menor medida. Su contribución en la intercepción de neblina fue del $22.6 \%, 8.5 \%$ y $11.6 \%$ en el colector CFC, SFC35 y SFC50, respectivamente. Del total de neblina interceptada por los colectores, la neblina captada sin la existencia de precipitación representó el $75.7 \%$ en el colector CFC, el $98.2 \%$ en el colector SFC35 y el $95.7 \%$ en el colector SFC50. Claramente la precipitación no tiene influencia en la intercepción de neblina de los colectores estándar, pero sí la tiene en el colector cilíndrico. Al ver el gráfico de caja-bigote (Figura 8), se puede evidenciar que la intensidad de intercepción de neblina del colector CFC no está influencia por la intensidad de precipitación, y que en este colector se llega interceptar neblina con las mayores intensidades de precipitación (0.3 y 0.4 $\mathrm{mm} / \mathrm{h}$ ). Aunque el porcentaje de neblina interceptaba con intensidades mayores a $0.2 \mathrm{~mm} / \mathrm{h}$ es de apenas el $12 \%$.

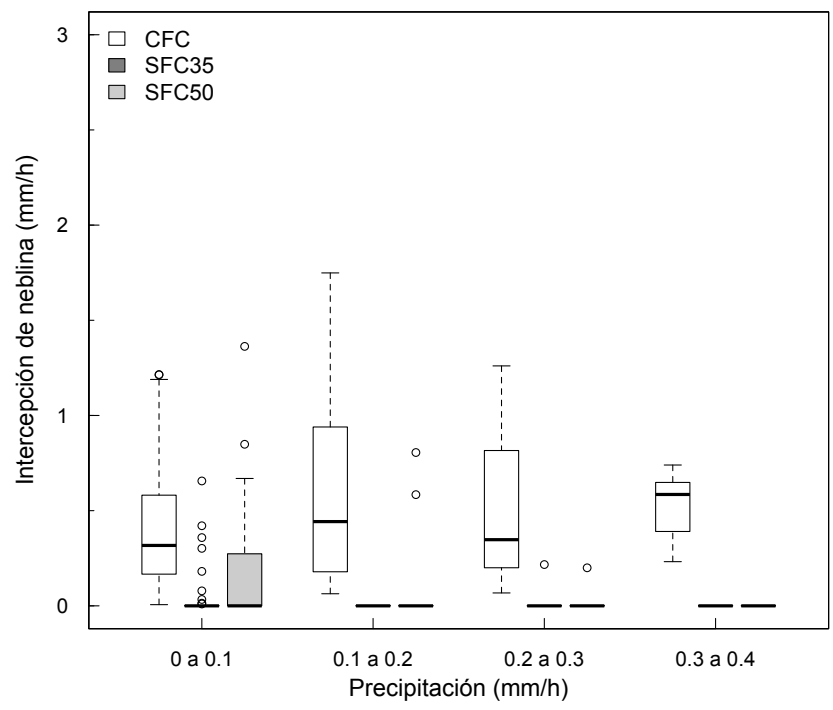

Figura 8. Estadísticas de la intensidad de intercepción de neblina en cada colector clasificadas por intervalos de precipitación durante el periodo de estudio (Junio-Julio 2016)

Variables como la temperatura y la humedad relativa presentan una contribución pequeña en la intercepción de neblina (con- 
tribución combinada menor del $8.2 \%$ en el mejor de los casos que corresponde al colector SFC50). Sin embargo, esto no quiere decir que su monitoreo no sea importante o que las variables no tengan realmente influencia. Ambas variables están relacionadas con el contenido de agua en el aire de la neblina. La humedad relativa indica el grado de saturación del aire, por lo que mientras mayor es la humedad relativa, mayor es el contenido de agua en el aire. La temperatura también tiene influencia en esta variable ya que el contenido de agua en el aire de la neblina está inversamente relacionado con el calor latente de condensación de la temperatura del agua [86]. Dominguez et al., 2017 [43] al estudiar la intercepción de neblina por parte de la vegetación endémica de San Cristóbal, compararon la intercepción de neblina por parte de la vegetación de dos temporadas de garúa distintas, y evidenciaron una diferencia significante, que dadas las condiciones (existió una diferencia significativa en la temperatura de las dos temporadas producto de una anomalía en la temperatura de la superficie del mar por el efecto temprano del evento de El Niño en la región $1+2$ ), la atribuyeron a una diferencia en el contenido de agua en el aire de la neblina.

En este caso de estudio, el monitoreo de la temperatura y la humedad relativa fue de dos meses durante la temporada de garúa, en donde las condiciones fueron relativamente constantes y es por esto que las dos variables no tuvieron una gran influencia en la intercepción de neblina por parte de los colectores. Posiblemente con un monitoreo más largo, que incluya la temporada cálida de invierno, se podría ver una mayor influencia de estas variables.

\section{B. Comparación de colectores}

El colector que presenta la mayor eficiencia de captación de neblina es el SFC50. Su intensidad de intercepción de neblina durante el periodo de estudio $(4.8 \mathrm{~mm} /$ día $)$ es 1.31 veces mayor que el colector CFC y 1.18 veces mayor que el colector SFC50. En el caso de captación de precipitación y neblina, también presenta un mayor rendimiento. El colector CFC presenta la peor eficiencia para captación de neblina. Esto puede ser explicado al repasar la dinámica del flujo de las gotas de agua de neblina que atraviesan 
una malla. La eficiencia de colección de neblina de un colector depende de su eficiencia aerodinámica, eficiencia de impactación y eficiencia de drenaje [61], [87]-[89]. La eficiencia de aerodinámica está relacionada a la capacidad que tiene la malla de dejar pasar el flujo de aire con neblina a través del mismo sin perturbar su curso. La eficiencia de impactación depende de la capacidad que tiene la superficie de la malla en atrapar las gotas de agua en la neblina que atraviesa la misma. La eficiencia de drenaje se refiere la capacidad que tiene la malla en drenar el agua de neblina captada por la malla rápidamente. La eficiencia total de un colector va a depender de la interacción de estas tres eficiencias. El colector CFC al ser cilíndrico, tiene una superficie aerodinámica que ayuda al paso lateral del flujo de aire con neblina, por lo que una parte del flujo en vez de atravesar el colector pasa rodeando su superficie y por lo tanto reduciendo su capacidad de colectar neblina. Además, su coeficiente de sombra relativamente alto, su pequeño tamaño de las aperturas de la malla y la forma cruzada de sus elementos, a pesar de mejorar la eficiencia de impactación (al existir una mayor área de obstrucción) producen un efecto adverso en la intercepción de neblina, las gotitas de agua que se interceptan bloquean el flujo de aire a través de los agujeros de la malla volviéndola más impenetrable [61]concomitantly with a reduction in ambient temperature and an increase in wind velocity ( $u$, produciendo que una mayor parte del flujo de aire no atraviese la malla y se desvíe. Este flujo que es desviado además de no llevar las gotas de agua a la malla puede acarrear las gotas que se encuentran en la malla y sacarlas fuera del área de colección. Esta situación podría empeorarse debido a la forma de la malla, que al tener fibras horizontales a poco distancia, la eficiencia de drenaje es reducida [88]. Este conjunto de factores podría explicar el bajo rendimiento del colector CFC en comparación de los otros colectores y además el por qué la intercepción de neblina en este colector es influenciado por la precipitación. En los eventos de neblina y precipitación, probablemente la lluvia, al tener un diámetro de gotas más grandes, choca con la malla y coalesce con las gotitas de agua de neblina interceptadas por la malla, mejorando el drenaje como consecuencia de su mayor tamaño. 
Los dos colectores SFC tienen las mismas dimensiones y la misma estructura. La única diferencia es su coeficiente de sombra. El SFC35, al tener más aberturas que el SFC50 permite el flujo del aire con neblina a través de su malla más fácilmente reduciendo la resistencia aerodinámica. Por el contrario, SFC35 tiene una menor área de impactación reduciendo la posibilidad de captar gotas de neblina en cuyo caso SFC50 sería más eficiente. Determinar la eficiencia de impactación y drenaje de estos colectores resulta en una serie de ecuaciones que dependen de varias variables y cuya determinación no entran en el alcance de este estudio (se recomienda leer Regalado \& Ritter, 2016 [88] para revisar estas ecuaciones). Sin embargo, la eficiencia aerodinámica puede ser determinada de forma teórica. Rivera, 2011 [87] utilizó un principio de superposición para separar el flujo de aire que elude un colector de neblina teórico impermeable y el flujo que pasa libremente a través de los agujeros de un colector de neblina. A partir de estas relaciones obtuvo el siguiente modelo para determinar la eficiencia aerodinámica:

$$
\eta_{A}=\frac{S C}{1+\sqrt{C_{O} / C_{D}}}(11),
$$

donde $S C$ es el coeficiente de sombra de la malla, $C_{D}$ es el coeficiente de arrastre y $C_{O}$ es el coeficiente de caída de presión. El coeficiente de arrastre depende del número de Reynolds y para flujo turbulento (esperado en condiciones típicas de neblina), $C$ tiene valores desde 1 para colectores cilíndricos hasta 2 para colectores cóncavos [87], [88]. Colectores de forma cuadrada alcanzan el valor de 1.18 [87], [90]. Co resulta de la relación de presiones entre el lado barlovento y sotavento del colector. Para el caso de una malla de seda (lo más parecido al material plástico o polietileno), puede ser determinado por [87]:

$$
C_{O}=1.62\left(1.3 S C+\left(\frac{S C}{1-S C}\right)^{2}\right)(12)
$$

siendo $C_{O}$ únicamente dependiente del coeficiente de sombra. De acuerdo a estas fórmulas, la eficiencia aerodinámica del colector CFC, SFC35 y SCF50 es de 0.16, 0.17 y 0.2, respectivamente. La eficiencia aerodinámica del colector SFC50 es la mayor, coincidiendo con los resultados encontrados en este estudio. La relación de las 
eficiencias aerodinámicas entre colectores es similar a la relación del rendimiento de captación de neblina entre colectores (Tabla 7).

Tabla 7. Relación de rendimiento experimental y eficiencia aerodinámica teórica entre colecotres

\begin{tabular}{|c|c|c|}
\hline Relación & Rendimiento experimental $^{\mathbf{a}}$ & Eficiencia teórica $^{\mathbf{b}}$ \\
\hline SCF50/SFC35 & 1.18 & 1.15 \\
\hline SCF50/CFC & 1.31 & 1.25 \\
\hline SFC35/CFC & 1.11 & 1.09 \\
\hline
\end{tabular}

a. Rendimiento de cada colector estimado a partir de la intercepción de neblina total registrada en el periodo de estudio.

b. Eficiencia teórica de cada colectore estimada a partir de la ecuación 11.

De acuerdo a la literatura [82], [87], [88] el colector de 50\% de coeficiente de sombra tiene una mayor eficiencia que el colector de 35\%. De acuerdo al modelo usado (Figura 9), las mejores eficiencias aerodinámicas se presentan entre valores de 50 y $60 \%$ de coeficiente de sombra [87]. Estos valores coinciden con las conclusiones de Schemenauer \& Joe, 1989 [82] en un estudio experimental en el norte de Chile, en donde usó una doble capa de malla Raschel de $35 \%$ de coeficiente de sombra (que resulta en valores aproximados al $50 \%$ de coeficiente de sombra).



Figura 9. Variación de la eficiencia aerodinámica en función del coeficiente de sombra de un colector (ecuación 11) 


\section{Comparación con otros sitos}

En San Cristóbal el colector SFC50 tuvo un rendimiento de captura de neblina y precipitación de $8.5 \mathrm{l} / \mathrm{m}^{2} \cdot$ día durante dos meses de la época de garúa. Este valor se encuentra por encima del promedio mundial (Figura 10).

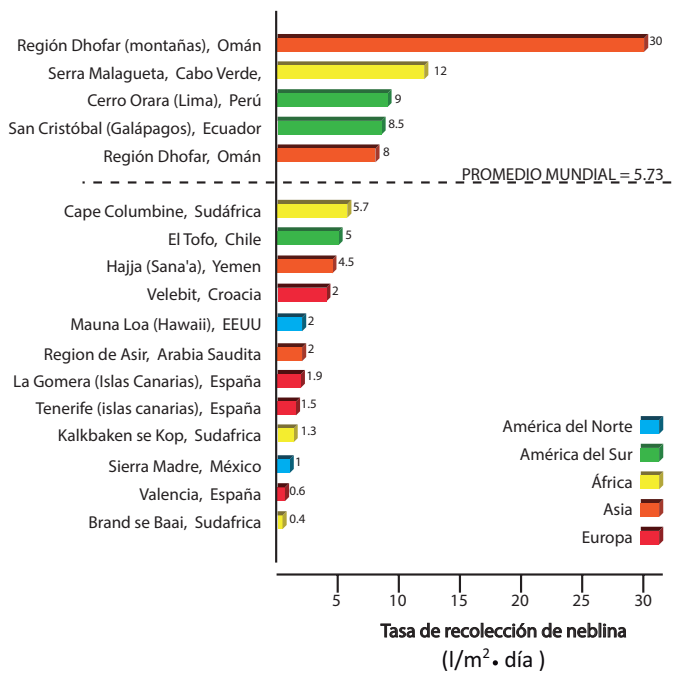

Figura 10. Tasa de intercepción de neblina en varios sitios a nivel global

Se han reportado rendimientos de 3 y $91 / \mathrm{m}^{2} \cdot$ día, en Chile y Perú, respectivamente, durante la época de neblina utilizando colectores de neblina estándar [45]. En un estudio en la zona del Caribe sudamericano (Colombia y Venezuela), Cavelier \& Goldstein, 1989 [37] realizaron un estudio sobre la intercepción de neblina encontrando que la intercepción de neblina varía entre 0.2 y 2.2 $\mathrm{mm}$ /día e identificaron que la intercepción de neblina sigue un gradiente orográfico en esta zona. En Sierra Madre, México, se registran valores entre 0.4 y $1.6 \mathrm{l} / \mathrm{m}^{2} \cdot$ día a elevaciones de 1330 a 1900 msnm [91]. Mientras que la recolección en Mauna Loa en Hawaii, a 2530 y $1580 \mathrm{msnm}$, alcanza valores de 1.9 y $2.1 \mathrm{~mm} /$ día utilizando un colector cilíndrico tipo Juvik [56]. En Asia, en regiones áridas, se han realizado mediciones de intercepción de neblina con varios propósitos. En Omán, Yemen y Arabia Saudita la recolección 
de neblina ha sido de $8,4.5$ y $21 / \mathrm{m}^{2}$.día, respectivamente [45], [82], [92]. En las montañas de la región de Dhofar (Omán), en una elevación entre 900 y 1000 msnm, se registró una alta recolección de neblina con un promedio de $30 \mathrm{l} / \mathrm{m}^{2}$.día durante tres meses de la temporada del monzón [45]. En el caso de Europa, se han realizado estudios principalmente en las islas Canarias, encontrando valores entre 0.2 y $5.0 \mathrm{l} / \mathrm{m}^{2}$.día en la isla La Gomera [54] y valores de 1.3 a $1.8 \mathrm{l} / \mathrm{m}^{2}$.día en Tenerife [52], [61]Taborno (1015 m a.s.l.. En la región Mediterránea de Valencia, se registró valores máximos de recolección de neblina hasta $7 \mathrm{l} / \mathrm{m}^{2}$.día [51]mientras que en Croacia en la montaña Velebit, la intercepción de neblina alcanzó valores hasta de $41 / \mathrm{m}^{2}$ ·día [93]. En África, la recolección de neblina también ha sido relevante. Sitios como la costa oeste de Sudáfrica, donde se han recolectado $0.4 \mathrm{l} / \mathrm{m}^{2}$.día y $1.3 \mathrm{l} / \mathrm{m}^{2}$.día, en Brand se Baai y Kalkbaken se Kop, respectivamente [94]. En éste último, la recolección de neblina es mayor y se atribuye a que se encuentra a más altura y además la velocidad del viento es mayor, siendo de 5.2 $\mathrm{m} / \mathrm{s}$ en promedio, mientras que en Brand se Baai es de $4.3 \mathrm{~m} / \mathrm{s}$. En Cape Columbine el promedio de recolección de agua es de $5.7 \mathrm{l} /$ $\mathrm{m}^{2}$ ·día [94]. En Cabo Verde, se reportó un promedio de recolección de neblina de $125 \mathrm{l} / \mathrm{m}^{2}$ ·día [95].

\section{Implementación del sistema de colección de neblina}

De acuerdo a los resultados de este estudio, se pudo evidenciar que tanto los colectores estándar como el colector cilíndrico son propicios para la captura de neblina en el Archipiélago. Dado que la dirección del viento no sufre variaciones drásticas y que los colectores estándar tienen un mejor rendimiento, se recomendaría el uso de colectores estándar, de preferencia colectores SFC50. Echeverría, 2018 [96] estimó la demanda de agua para consumo doméstico de la zona rural de San Cristóbal, obteniendo un valor de $80.3 \mathrm{l}$ / día-habitante. Para el caso de fincas, la autora estimó que una familia promedio se compone de 5 personas, por lo que una finca tendría una demanda de 401.5 l/día. Esto quiere decir que de acuerdo a los resultados de este estudio, durante la época seca, colectores de neblina con una malla raschell de $50 \%$ de coeficiente de sombra con 
un área de intercepción de $48 \mathrm{~m}^{2}$ ubicados a una elevación de 600 m.s.n.m. podrían cumplir $100 \%$ de la demanda (para elevaciones más bajas se necesitaría un área mayor de recolección). Utilizando un sistema de colección de aguas lluvias en el techo con un área de $200 \mathrm{~m} 2$ se podría colectar 0.3 1/día. Existen varias comunidades que han utilizado la cosecha de neblina como una fuente de agua para su población. Chungungo, una comunidad ubicada en la zona desértica de la costa de Chile utiliza un sistema de cosecha de neblina de 100 colectores grandes $(10 \mathrm{x} 4 \mathrm{~m})$, produciendo en promedio 15000 1 de agua potable para 300 habitantes de la villa [32]. De acuerdo a Batisha, 2015 [15], otros proyectos de colección de neblina están produciendo 6300 1/día en Tojquia (Guatemala) con $1400 \mathrm{~m} 2$ de superficie de colección y cantidades viables en sitios como Sudáfrica, Perú y Colombia.

Los sistemas de colección de neblina además de ser amigables con el medio ambiente (no requieren energía eléctrica), sostenibles en el tiempo (bajo costo operacional) también resultan ser relativamente baratos comparados con otros sistemas convencionales que requiere una inversión inicial grande, costos operacionales altos y un alto mantenimiento [32].

En Chile, un sistema de cosecha de neblina puede ser producido por cerca de $\$ 1$ por $\mathrm{m}^{3}$ y transportado a los hogares de una comunidad lejana por $\$ 1$ por $\mathrm{m}^{3}$ [44]. Batisha, 2015 comenta que el costo de 100 colectores de neblina grandes, adecuado para una pequeña comunidad, estaría en el orden de $\$ 40000$, que es barato comparado con otros sistemas de agua. Claramente, este sistema alternativo de agua tiene un potencial alto. Ciudades y comunidades inteligentes, deben tomar en cuenta sistemas de agua sustentables y amigables con el ambiente como es el caso de la cosecha de neblina.

\section{Conclusiones}

La parte alta de la isla San Cristóbal (Galápagos) está cubierta por una capa de neblina casi permanente durante 7 meses al año. Aprovechando este fenómeno, durante dos meses se ha instalado colectores de neblina para cuantificar la intercepción de neblina y evaluar su potencial como una fuente de agua alternativa para las 
comunidades del sector. Se usaron tres colectores de neblina, uno cilíndrico y dos colectores estándar bidimensionales de 35 y 50\% de coeficiente de sombra. Las mediciones fueron complementadas con el monitoreo de las principales variables meteorológicas.

Los resultados muestran que el rendimiento de los colectores es de 3.8, 7.0 y $8.5 \mathrm{l} / \mathrm{m}^{2}$ día en el colector CFC, SFC35 y SCF50, respectivamente. Utilizando un modelo geométrico, se determinó la profundidad de intercepción de neblina únicamente (separado de la precipitación), obteniéndose valores de $176.5 \mathrm{~mm}$ en el colector CFC, $195.8 \mathrm{~mm}$ en el colector SFC35 y 230.6 en el colector SFC50. Estos valores corresponden a más del $242 \%$ de la lluvia, lo que evidencia el potencial de intercepción de neblina en el sector. Así mismo, el rendimiento del colector SFC50 está por encima del promedio de intercepción de neblina mundial.

Al igual que en otros estudios, se determinó que la velocidad del viento es la variable que más influencia tiene sobre la intercepción de neblina (aprox. 50\%), seguida de la radiación solar y de la lluvia. Esta última tiene una mayor influencia en el colector CFC. El hecho de que la intercepción de neblina incremente con la velocidad del viento y no se estanque en una meseta indica el potencial de neblina de sector, incluso con velocidades del viento más fuertes o un contenido de agua en el aire más bajo. La dirección del viento no tuvo influencia sobre la intercepción de neblina porque permaneció relativamente constante durante el periodo de estudio, lo que justifica el uso de colectores bidimensionales en vez de colectores cilíndricos que tienen una menor eficiencia de recolección de neblina.

El colector SFC50 resultó ser el que mayor eficiencia de intercepción de neblina tiene. Esto se puede explicar por su coeficiente de sombra, el cual permite una mayor área de obstrucción para interceptar las gotas de agua en el flujo de aire que atraviesa su malla sin causar demasiadas obstrucciones que obliguen al flujo de aire desviarse de su trayectoria de intercepción con la malla. El colector CFC es el que menor eficiencia tiene, debido a su forma aerodinámica permite el paso lateral del flujo del aire. Además, el pequeño tamaño de sus aberturas induce a la obstrucción de las mismas con gotas de agua, haciendo que la superficie sea menos permeable y por lo tanto se capte menor neblina. Se concluye que la influencia de la lluvia en 
la intercepción de neblina en este colector es debido a que ayuda al drenaje rápido de la neblina interceptada en la malla.

El potencial de cosecha de neblina en Galápagos como una fuente alternativa de agua resulta evidente. Este sistema podría ser implementado como una fuente alternativa de agua para este sector, sobre todo en las islas donde los recursos hídricos superficiales permanentes son escasos o inexistentes. Esta información es de vital importancia para las autoridades que trabajan en el manejo de los recursos hídricos en estas islas, sobre todo por el hecho de la escasez de fuentes superficiales de agua en este territorio. Así mismo, la metodología usada sirve para la evaluación de la implementación de un sistema de cosecha de neblina en otras partes del mundo. Claro que sería necesario complementar esta investigación con la evaluación de la calidad del agua interceptada de la neblina, una evaluación económica y el uso de otras tecnologías de intercepción de neblina (ej. material hidrofóbico [97]). En la planeación de desarrollar ciudades inteligentes, la sostenibilidad es un aspecto importante. Técnicas como la cosecha de neblina, entran dentro de esta idea de sostenibilidad.

\section{Agradecimientos}

El estudio se llevó a cabo dentro del marco del proyecto Galápagos Islands Integrated Water Studies (GIIWS). El equipo GIIWS quisiera agradecer a la Agence Nationale de la Recherche (ANR-blanc 2010 GIIWS Ref.601-01) y Galapagos Conservancy por su financiamiento. También quisiéramos agradecer a nuestros colaboradores locales en Galápagos: la Estación de Investigación Charles Darwin, el Parque Nacional Galápagos, y propietarios privados que permitieron la instalación de las estaciones climáticas.

\section{Referencias}

[1] V. Albino, U. Berardi, and R. M. Dangelico, "Smart Cities: Definitions, Dimensions, Performance, and Initiatives," J. Urban Technol., vol. 22, no. 1, pp. 3-21, Jan. 2015.

[2] T. Bakıc1, E. Almirall, and J. Wareham, "A Smart City Initiative: the Case of Barcelona," J. Knowl. Econ., vol. 4, no. 2, pp. 135-148, 2013. 
[3] T. M. Chen, "Smart grids, smart cities need better networks [Editor's Note]," IEEE Netw., vol. 24, no. 2, pp. 2-3, 2010.

[4] L.-G. Cretu, "Smart Cities Design using Event-driven Paradigm and Semantic Web," Inform. Econ., vol. 16, no. 4, pp. 57-67, 2012.

[5] C. Harrison et al., "Foundations for Smarter Cities," IBM J. Res. Dev., vol. 54, no. 4, pp. 1-16, 2010.

[6] P. Lombardi, S. Giordano, H. Farouh, and W. Yousef, "Modelling the smart city performance," Innov. Eur. J. Soc. Sci. Res., vol. 25, no. 2, pp. 137-149, Jun. 2012.

[7] G. C. Lazaroiu and M. Roscia, "Definition methodology for the smart cities model," Energy, vol. 47, no. 1, pp. 326-332, 2012.

[8] L. Guan, "Smart Steps To A Battery City," Goverment news, vol. 32, no. 2, pp. 24-27, 2012.

[9] N. Komninos, "Intelligent cities: Variable geometries of spatial intelligence," Intell. Build. Int., vol. 3, no. 3, pp. 172-188, Jul. 2011.

[10] K. Kourtit, P. Nijkamp, and D. Arribas, "Smart cities in perspective - a comparative European study by means of self-organizing maps," Innov. Eur. J. Soc. Sci. Res., vol. 25, no. 2, pp. 229-246, Jun. 2012.

[11] M. Thuzar, "Urbanization in SouthEast Asia: Developing Smart Cities for the Future," Reg. Outlook, pp. 96-100, 2011.

[12] A. Caragliu, C. Del Bo, and P. Nijkamp, "Smart Cities in Europe," J. Urban Technol., vol. 18, no. 2, pp. 65-82, Apr. 2011.

[13] T. Nam and T. A. Pardo, "Conceptualizing Smart City with Dimensions of Technology, People, and Institutions," in Proceedings of the 12th Annual International Digital Government Research Conference: Digital Government Innovation in Challenging Times, 2011, pp. 282-291.

[14] S. Zygiaris, "Smart City Reference Model: Assisting Planners to Conceptualize the Building of Smart City Innovation Ecosystems," J. Knowl. Econ., vol. 4, no. 2, pp. 217-231, 2013.

[15] A. F. Batisha, "Feasibility and sustainability of fog harvesting," Sustain. Water Qual. Ecol., vol. 6, pp. 1-10, 2015.

[16] L. Junguo et al., "Water scarcity assessments in the past, present, and future," Earth's Futur., vol. 5, no. 6, pp. 545-559, Mar. 2017.

[17] F. R. Rijsberman, "Water scarcity: Fact or fiction?," Agric. Water Manag., vol. 80, no. 1, pp. 5-22, 2006.

[18] J. Schewe et al., "Multimodel assessment of water scarcity under climate change," Proc. Natl. Acad. Sci., vol. 111, no. 9, p. 3245 LP-3250, Mar. 2014.

[19] N. W. Arnell, "Climate change and global water resources:SRES emissions and socio-economic scenarios," Glob. Environ. Chang., vol. 14, no. 1, pp. 31-52, 2004.

[20] T. Oki and S. Kanae, "Global Hydrological Cycles and World Water Resources," Science (80-. )., vol. 313, no. 5790, p. 1068 LP-1072, Aug. 2006. 
[21] J. ALCAMO, M. FLÖRKE, and M. MÄRKER, "Future long-term changes in global water resources driven by socio-economic and climatic changes," Hydrol. Sci. J., vol. 52, no. 2, pp. 247-275, Apr. 2007.

[22] A. Hayashi, K. Akimoto, F. Sano, S. Mori, and T. Tomoda, "Evaluation of global warming impacts for different levels of stabilization as a step toward determination of the long-term stabilization target," Clim. Change, vol. 98, no. 1, p. 87, 2009.

[23] N. W. Arnell and S. N. Gosling, "The impacts of climate change on river flow regimes at the global scale," J. Hydrol., vol. 486, pp. 351-364, 2013.

[24] S. N. Gosling and N. W. Arnell, "A global assessment of the impact of climate change on water scarcity," Clim. Change, vol. 134, no. 3, pp. 371-385, 2016.

[25] C. J. Vörösmarty, P. Green, J. Salisbury, and R. B. Lammers, "Global Water Resources: Vulnerability from Climate Change and Population Growth," Science (80-. )., vol. 289, no. 5477, p. 284 LP-288, Jul. 2000.

[26] S. Dolnicar and A. Hurlimann, "Drinking water from alternative water sources: differences in beliefs, social norms and factors of perceived behavioural control across eight Australian locations," Water Sci. Technol., vol. 60, no. 6, p. 1433 LP-1444, Sep. 2009.

[27] S. Piao et al., "The impacts of climate change on water resources and agriculture in China," Nature, vol. 467, p. 43, Sep. 2010.

[28] R. G. Raluy, L. Serra, and J. Uche, "Life Cycle Assessment of Water Production Technologies - Part 1: Life Cycle Assessment of Different Commercial Desalination Technologies (MSF, MED, RO) (9 pp)," Int. J. Life Cycle Assess., vol. 10, no. 4, pp. 285-293, 2005.

[29] R. G. Raluy, L. Serra, J. Uche, and A. Valero, "Life Cycle Assessment of Water Production Technologies - Part 2: Reverse Osmosis Desalination versus the Ebro River Water Transfer (9 pp)," Int. J. Life Cycle Assess., vol. 10, no. 5, pp. 346-354, 2005.

[30] J. Stokes and A. Horvath, "Life Cycle Energy Assessment of Alternative Water Supply Systems (9 pp)," Int. J. Life Cycle Assess., vol. 11, no. 5, pp. 335343, 2006.

[31] O. Klemm et al., "Fog as a Fresh-Water Resource: Overview and Perspectives," Ambio, vol. 41, no. 3, pp. 221-234, 2012.

[32] M. Fessehaye, S. A. Abdul-Wahab, M. J. Savage, T. Kohler, T. Gherezghiher, and H. Hurni, "Fog-water collection for community use," Renew. Sustain. Energy Rev., vol. 29, pp. 52-62, 2014.

[33] V. J. Camilo, T. Conrado, and B. D. D., "Fog interception by non-vascular epiphytes in tropical montane cloud forests: dependencies on gauge type and meteorological conditions," Hydrol. Process., vol. 22, no. 14, pp. 2484-2492, Jun. 2008.

[34] R. J. Pilié, E. J. Mack, C. W. Rogers, U. Katz, and W. C. Kocmond, "The Formation of Marine Fog and the Development of Fog-Stratus Systems along the California Coast," J. Appl. Meteorol., vol. 18, no. 10, pp. 1275-1286, Oct. 1979. 
[35] S. B. LA, E. Werner, and B. Reto, "Fog as a Hydrologic Input," Encyclopedia of Hydrological Sciences. 15-Apr-2006.

[36] S. Prada, M. Menezes de Sequeira, C. Figueira, and M. O. da Silva, "Fog precipitation and rainfall interception in the natural forests of Madeira Island (Portugal)," Agric. For. Meteorol., vol. 149, no. 6, pp. 1179-1187, 2009.

[37] J. Cavelier and G. Goldstein, "Mist and fog interception in elfin cloud forests in Colombia and Venezuela," J. Trop. Ecol., vol. 5, no. 03, pp. 309-322, 1989.

[38] L. B. Hutley, D. Doley, D. J. Yates, and A. Boonsaner, "Water Balance of an Australian Subtropical Rainforest at Altitude: the Ecological and Physiological Significance of Intercepted Cloud and Fog," Aust. J. Bot., vol. 45, no. 2, p. 311, 1997.

[39] D. McJannet, J. Wallace, and P. Reddell, "Precipitation interception in Australian tropical rainforests: 2. Altitudinal gradients of cloud interception, stemflow, throughfall and interception," Hydrol. Process., vol. 21, no. 13, pp. 1703-1718, 2007.

[40] F. Holwerda, R. Burkard, W. Eugster, F. N. Scatena, A. G. C. A. Meesters, and L. A. Bruijnzeel, "Estimating fog deposition at a Puerto Rican elfin cloud forest site: comparison of the water budget and eddy covariance methods," Hydrol. Process., vol. 20, no. 13, pp. 2669-2692, 2006.

[41] L. A. Bruijnzeel, M. Mulligan, and F. N. Scatena, "Hydrometeorology of tropical montane cloud forests: emerging patterns: Hydrometeorology of tropical montane cloud forests," Hydrol. Process., vol. 25, no. 3, pp. 465-498, 2011.

[42] Y. Uehara and A. Kume, "Canopy Rainfall Interception and Fog Capture by Pinus pumila Regal at Mt. Tateyama in the Northern Japan Alps, Japan," Arctic, Antarct. Alp. Res., vol. 44, no. 1, pp. 143-150, 2012.

[43] C. Domínguez et al., "Quantification of cloud water interception in the canopy vegetation from fog gauge measurements," Hydrol. Process., vol. 31, no. 18, pp. 3191-3205, Aug. 2017.

[44] P. Cereceda, R. S. Schemenauer, and M. Suit, "An alternative water supply for chilean coastal desert villages," Int. J. Water Resour. Dev., vol. 8, no. 1, pp. 53-59, Mar. 1992.

[45] R. S. Schemenauer and P. Cereceda, "A Proposed Standard Fog Collector for Use in High-Elevation Regions," J. Appl. Meteorol., vol. 33, no. 11, pp. 1313-1322, Nov. 1994.

[46] E. C. David and R. W. Zimmerman, "Compressibility and shear compliance of spheroidal pores: Exact derivation via the Eshelby tensor, and asymptotic expressions in limiting cases," Int. J. Solids Struct., vol. 48, no. 5, pp. 680-686, 2011.

[47] E. S. Shanyengana, J. R. Henschel, M. K. Seely, and R. D. Sanderson, "Exploring fog as a supplementary water source in Namibia," Atmos. Res., vol. 64, no. 1, pp. 251-259, 2002.

[48] M. V. Marzol Jaén, "Fog water collection in a rural park in the Canary Islands (Spain)," Atmos. Res., vol. 64, no. 1, pp. 239-250, 2002. 
[49] J. Olivier, "Fog harvesting: An alternative source of water supply on the West Coast of South Africa," GeoJournal, vol. 61, no. 2, p. 203, 2004.

[50] S. A. Abdul-Wahab, A. M. Al-Damkhi, H. Al-Hinai, K. A. Al-Najar, and M. S. Al-Kalbani, "Total fog and rainwater collection in the Dhofar region of the Sultanate of Oman during the monsoon season," Water Int., vol. 35, no. 1, pp. 100-109, Feb. 2010.

[51] M. J. Estrela, J. A. Valiente, D. Corell, and M. M. Millán, "Fog collection in the western Mediterranean basin (Valencia region, Spain)," Atmos. Res., vol. 87, no. 3, pp. 324-337, 2008.

[52] A. Ritter, M. C. Regalado, and C. J. Guerra, "Quantification of Fog Water Collection in Three Locations of Tenerife (Canary Islands)," Water , vol. 7, no. 7. 2015.

[53] K. A. Brauman, D. L. Freyberg, and G. C. Daily, "Forest structure influences on rainfall partitioning and cloud interception: A comparison of native forest sites in Kona, Hawai'i," Agric. For. Meteorol., vol. 150, no. 2, pp. 265-275, 2010.

[54] A. Ritter, C. M. Regalado, and G. Aschan, "Fog Water Collection in a Subtropical Elfin Laurel Forest of the Garajonay National Park (Canary Islands): A Combined Approach Using Artificial Fog Catchers and a Physically Based Impaction Model," J. Hydrometeorol., vol. 9, no. 5, pp. 920-935, 2008.

[55] E. Gioda, G. R. Espejo, and A. Acosta Baladón, "Fog collectors in tropical areas," in Proceedings of the symposium on precipitation and evaporation, 1993, pp. 4-20.

[56] E. P. C. Juvik J.O., "A climatology of mountain fog on Mauna Loa, Hawai'i Island," Univ. Hawai'i Water Resour. Cent. Tech. Rep., no. 118, 1978.

[57] K. F. A. Frumau, R. Burkard, S. Schmid, L. A. (Sampurno) Bruijnzeel, C. Tobón, and J. C. Calvo-Alvarado, "A comparison of the performance of three types of passive fog gauges under conditions of wind-driven fog and precipitation," Hydrol. Process., vol. 25, no. 3, pp. 374-383, 2011.

[58] J. Van Heerden, J. Olivier, and L. van Schalkwyk, "Van Heerden, J., Olivier, J., van Schalkwyk, L., 2010. Fog water systems in South Africa: an update. In: Proceedings of the 5th International Conference on Fog, Fog Collection and Dew, July 25-30, 2010, Münster, p. 160.," In: Proceedings of the 5th International Conference on Fog, Fog Collection and Dew, 2010, p. 160.

[59] P. Cereceda, P. Hernández, J. Leiva, and J. Rivera, "Agua de Niebla," Santiago: Pontificia Universidad Católica, 2014.

[60] K. J. Tiedemann and A. Lummerich, "Fog harvesting on the verge of economic competitiveness," in In: Proceedings of the 5th International Conference on Fog, Fog Collection and Dew, 2010, p. 192.

[61] C. M. Regalado and A. Ritter, "The performance of three fog gauges under field conditions and its relationship with meteorological variables in an exposed site in Tenerife (Canary Islands)," Agric. For. Meteorol., vol. 233, pp. 80-91, 2017. 
[62] C. Tomasi and F. Tampieri, "Features of the proportionality coefficient in the relationship between visibility and liquid water content in haze and fog," Atmosphere (Basel)., vol. 14, no. 2, pp. 61-76, Jun. 1976.

[63] K. F. A. Frumau, L. A. Bruijnzeel, C. Tobón, and others, "Hydrological measurement protocol for montane cloud forests. Annex 2, Final Technical Report on DFID-FRP Project no. R7991.," 2006.

[64] R. Gunn and G. D. Kinzer, "The terminal velocity of fall for water droplets in stagnant air," J. Meteorol., vol. 6, no. 4, pp. 243-248, 1949.

[65] S. R. Herwitz, "Interception storage capacities of tropical rainforest canopy trees," J. Hydrol., vol. 77, no. 1-4, pp. 237-252, 1985.

[66] INEC, "Resultados del censo 2010 de población y vivienda en el Ecuador," Quito, Ecuador, 2010.

[67] E. C. and T. A., "The influence of the Galápagos Islands on tropical temperatures, currents and the generation of tropical instability waves," Geophys. Res. Lett., vol. 31, no. 15, Aug. 2004.

[68] M. Trueman and N. D'Ozouville, "Characterizing the Galapagos terrestrial climate in the face of global climate change," Galapagos Res., vol. 67, pp. 26-37, 2010.

[69] A. Pryet et al., "Quantification of cloud water interception along the windward slope of Santa Cruz Island, Galapagos (Ecuador)," Agric. For. Meteorol., vol. 161, pp. 94-106, 2012.

[70] R. B. Dunbar, G. M. Wellington, M. W. Colgan, and P. W. Glynn, "Eastern Pacific sea surface temperature since 1600 A.D.: The record of climate variability in Galápagos Corals," Paleoceanography, vol. 9, no. 2, pp. 291-315, 1994.

[71] C. G. Domínguez et al., "Comparison of deep percolation rates below contrasting land covers with a joint canopy and soil model," J. Hydrol., vol. 532, pp. 65-79, 2016.

[72] D. J. Geist, A. R. McBIRNEY, and R. A. Duncan, "Geology and petrogenesis of lavas from San Cristobal Island, Galapagos Archipelago," Geol. Soc. Am. Bull., vol. 97, no. 5, p. 555, 1986.

[73] M. Adelinet, J. Fortin, N. D’Ozouville, and S. Violette, "The relationship between hydrodynamic properties and weathering of soils derived from volcanic rocks, Galapagos Islands (Ecuador)," Environ. Geol., vol. 56, no. 1, pp. 45-58, 2008.

[74] A. Pryet, N. d'Ozouville, S. Violette, B. Deffontaines, and E. Auken, "Hydrogeological settings of a volcanic island (San Cristóbal, Galapagos) from joint interpretation of airborne electromagnetics and geomorphological observations," Hydrol. Earth Syst. Sci., vol. 16, no. 12, pp. 4571-4579, 2012.

[75] S. Violette, N. d'Ozouville, A. Pryet, B. Deffontaines, J. Fortin, and M. Adelinet, "Hydrogeology of the Galápagos Archipelago: An Integrated and Comparative Approach Between Islands," in Geophysical Monograph Series, K. S. Harpp, E. Mittelstaedt, N. d'Ozouville, and D. W. Graham, Eds. Hoboken, New Jersey: John Wiley \& Sons, Inc, 2014, pp. 167-183. 
[76] N. D'Ozouville et al., "Extensive perched aquifer and structural implications revealed by 3D resistivity mapping in a Galapagos volcano," Earth Planet. Sci. Lett., vol. 269, no. 3-4, pp. 518-522, May 2008.

[77] C. Domínguez, "Integrated Hydrogeological Study of San Cristobal Island (Galapagos)," Université Pierre et Marie Curie - Paris VI, Paris, 2016.

[78] M. Adelinet, "Etude du fonctionnement hydrologique des bassins versant de San Cristobal (Galapagos - Equateur," Université Pierre et Marie Curie Paris $\{$ VI $\}$, Paris, 2005.

[79] N. d'Ozouville, "Etude du Fonctionnement Hydrologique Dans les Iles Galápagos : caractérisation d'un milieu volcanique insulaire et préalable à la gestion de la ressource.," 2007.

[80] (CGREG) Consejo de Gobierno del Régimen Especial de Galápagos, "Censo de Unidades de Producción Agropecuaria de Galápagos," 2014.

[81] D. and R. (CISPDR) Changjiang Institute of Survey, Planning, "La planificación de los recursos hídricos de las islas galápagos," 2015.

[82] R. S. Schemenauer and P. I. Joe, "The collection efficiency of a massive fog collector," Atmos. Res., vol. 24, no. 1, pp. 53-69, 1989.

[83] J. C. Villegas, C. Tobón, and D. D. Breshears, "Fog interception by non-vascular epiphytes in tropical montane cloud forests: dependencies on gauge type and meteorological conditions," Hydrol. Process., vol. 22, no. 14, pp. 2484-2492, 2008.

[84] W. Eugster, R. Burkard, F. Holwerda, F. N. Scatena, and L. A. S. Bruijnzeel, "Characteristics of fog and fogwater fluxes in a Puerto Rican elfin cloud forest," Agric. For. Meteorol., vol. 139, no. 3, pp. 288-306, 2006.

[85] T. W. Giambelluca and D. Nullet, "Influence of the trade-wind inversion on the climate of a leeward mountain slope in \{Hawaii\}," Clim. Res., vol. 1, no. 3, pp. 207-216, 1991.

[86] A. Thompson, "Simulating the adiabatic ascent of atmospheric air parcels using the cloud chamber," Dep. Meteorol. Penn State, 2007.

[87] J. de D. Rivera, "Aerodynamic collection efficiency of fog water collectors," Atmos. Res., vol. 102, no. 3, pp. 335-342, 2011.

[88] C. M. Regalado and A. Ritter, "The design of an optimal fog water collector: A theoretical analysis," Atmos. Res., vol. 178-179, pp. 45-54, 2016.

[89] D. M. and T. Fernandez Alicia and Weiss-Penzias, Peter S. and Zhang, Bong June and Sorensen, Deckard and Cohen, Robert E. and McKinley, Gareth $\mathrm{H}$. and Kleingartner, Justin and Oliphant, Andrew and Bowman, Matthew, "Fog Water Collection Effectiveness: Mesh Intercomparisons," Aerosol Air Qual. Res., vol. 18, no. 1, pp. 270-283, 2018.

[90] F. M. White, Fluid Mechanics, Second. New York: McGraw-Hill Book Company, 1986.

[91] H. W. Vogelmann, "Fog Precipitation in the Cloud Forests of Eastern Mexico," Bioscience, vol. 23, no. 2, pp. 96-100, Feb. 1973.

[92] G. A. Al-hassan, "Fog Water Collection Evaluation in Asir Region-Saudi Arabia," Water Resour. Manag., vol. 23, no. 13, pp. 2805-2813, 2009. 
[93] Z. Mileta, M., Likso, "Fog water collection with SFC on the mountain Velebit (Croatia) during the period 2000-2009," in Proceedings of the 5th International Conference on Fog, Fog Collection and Dew, July 25-30, 2010, 2010, pp. 240-242.

[94] J. Olivier, "Fog-water harvesting along the West Coast of South Africa: A feasibility study," Water SA, vol. 28, no. 4, pp. 349-360, 2002.

[95] A. Sabino, "Fog collection in the natural park of Serra Malagueta. An alternative source of water for the communities.," in Proceedings of the 4th international conference on fog, fog collection and dew, 2007, pp. 425-428.

[96] P. Echeverría, "potencial de la captura de niebla para uso doméstico rural y riego durante la época seca del año en la isla San Cristobal, Galápagos, Ecuador," Escuela Politécnica Nacional, Ecuador, 2018.

[97] M. Rajaram, X. Heng, M. Oza, and C. Luo, "Enhancement of fog-collection efficiency of a Raschel mesh using surface coatings and local geometric changes," Colloids Surfaces A Physicochem. Eng. Asp., vol. 508, pp. 218-229, 2016.

\section{Sobre los autores}

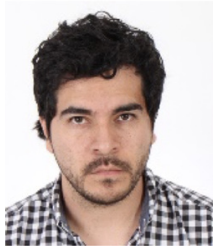

Christian Domínguez. (Y'1987-M'07) recibió el título de Ingeniero Civil de la Escuela Politécnica Nacional (2011) y el título de doctor en geociencia, recursos naturales y medio ambiente de la Universidad Pierre et Marie Curie (2016). Actualmente es profesor titular de la Carrera de Ingeniería Civil de la Universidad Politécnica Salesiana.

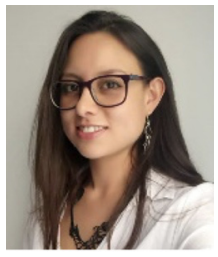

Paola Alejandra Echeverría Garcés (Y’1991-M’09). Paola Echeverría es Ingeniera Ambiental, obtuvo su título en la Facultad de Ingeniería Civil y Ambiental de la Escuela Politécnica Nacional en el año 2018.

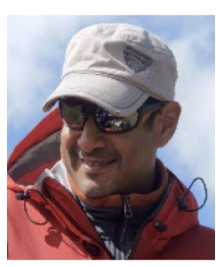

Marcos Villacís (Y'1975 - M’06) recibió el título de Ingeniero Civil de la Escuela Politécnica Nacional (2001), realizó una maestría en Hidrología e Hidrogeología en la Universidad Pierre et Marie Curie (2003) y tiene un PhD en Aguas Continentales y Sociedad en la Universidad Montpellier 2 (2008). Actualmente es profesor titular del departamento de Ingeniería Civil y Ambiental de la Escuela Politécnica Nacional (EPN). En el ámbito de gestión académica, fue decano 
(2015-2016) y jefe de departamento (2016-2018) de la Facultad Ingeniería Civil y Ambiental de la EPN. Su investigación se enfoca principalmente en la hidro-glaciología y el cambio climático, siendo co-director del proyecto de investigación "Linking Gobal Change with soil an water conservation in the high Andes" y co-director del Laboratorio Mixto Internacional GREATICE (Glaciares y Recursos Hídricos de Altura: Indicadores de Cambios Ambientales). En la actualidad, es editor invitado de la Revista Politécnica de la EPN y de la Revista Científica La Granja de la UPS.

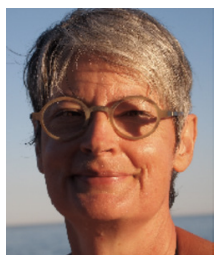

Sophie Violette (Y'1966) recibió su PhD (1993) y luego un HDR (2003) en Hidrogeología de la Universidad Pierre et Marie Curie (UPMC). Actualmente es investigadora en el Laboratorio de Geología UMR.8538 de la Escuela Normal Superior (Francia) y es profesora titular en Hidrogeología en la Universidad Sorbona desde 1995. Sophie trabajó como científica visitante en la Universidad John Hopkins dentro del equipo de Prof. Grant Garven (1999) y en la Universidad Stanford dentro del equipo del Prof. Steve Gorelick (2005). El principal objetivo de sus actividades de investigación es entender los flujos de aguas subterráneas a varias escalas espaciales y temporales a partir de la identificación de: geometría en 3D (datos geofísicos y geológicos), propiedades (físicas, mecánicas, hidrodinámicas, transferencia de solutos y de calor), procesos físicos envueltos y condiciones de borde (tectónica, clima y el bombeo de aguas subterráneas). Sus actividades científicas incluyen una larga colección de modelos de flujos hidrológicos y tiene antecedentes multidisciplinarios combinados con experiencia en campo en regiones con varios climas como: Francia (islas volcánicas y cuencas sedimentarias), Bulgaria y Madagascar (lechos rocosos), India (cuencas sedimentarias), Ecuador e Indonesia (volcanes basálticos y andesíticos). Su experticia ha sido fortalecida por su participación y dirección de proyectos de investigación con colaboradores nacionales e internaciones. Su investigación ha sido financiada por: organismos públicos (AESN, ANDRA, ANR, ANRT, Chancellery of Universities, CRIF, INSU, IRSN, SEDIF), compañías privadas (DANONE, EDF, ESA, GDF-Suez), fundaciones (Ensemble, Foundation of France, Schlumberger, Véolia) y organismos internacionales (PNUD, World bank). Ella ha servido como experta en hidrogeología en varios comités internaciones (ANDRA-COS, IFPEN-CS, Universities-CSE, INRA-CSS). 